Draft Version OCtOBer 25, 2018

Preprint typeset using $\mathrm{LAT}_{\mathrm{E}} \mathrm{X}$ style emulateapj v. 08/22/09

\title{
HALO OCCUPATION DISTRIBUTION MODELING OF CLUSTERING OF LUMINOUS RED GALAXIES
}

\author{
Zheng Zheng ${ }^{1,2,3}$, Idit Zehavi ${ }^{4}$, Daniel J. Eisenstein ${ }^{5}$, David H. Weinberg ${ }^{6}$, and Y.P. Jing ${ }^{7}$ \\ Draft version October 25, 2018
}

\begin{abstract}
We perform Halo Occupation Distribution (HOD) modeling to interpret small-scale and intermediate-scale clustering of 35,000 luminous early-type galaxies and their cross-correlation with a reference imaging sample of normal $L_{*}$ galaxies in the Sloan Digital Sky Survey. The modeling results show that most of these luminous red galaxies (LRGs) are central galaxies residing in massive halos of typical mass $M \sim$ a few times $10^{13}$ to $10^{14} h^{-1} M_{\odot}$, while a few percent of them have to be satellites within halos in order to produce the strong auto-correlations exhibited on smaller scales. The mean luminosity $L_{c}$ of central LRGs increases with the host halo mass, with a rough scaling relation of $L_{c} \propto M^{0.5}$. The halo mass required to host on average one satellite LRG above a luminosity threshold is found to be about 10 times higher than that required to host a central LRG above the same threshold. We find that in massive halos the distribution of $L_{*}$ galaxies roughly follows that of the dark matter and their mean occupation number scales with halo mass as $M^{1.5}$. The HOD modeling results also allows for an intuitive understanding of the scale-dependent luminosity dependence of the cross-correlation between LRGs and $L_{*}$ galaxies. Constraints on the LRG HOD provide tests to models of formation and evolution of massive galaxies, and they are also useful for cosmological parameter investigations. In one of the appendices, we provide LRG HOD parameters with dependence on cosmology inferred from modeling the two-point auto-correlation functions of LRGs.
\end{abstract}

Subject headings: cosmology: observations — galaxies: halos — galaxies: statistics — galaxies: clusters: general - galaxies: elliptical and lenticular, cD — galaxies: evolution

\section{INTRODUCTION}

The clustering of galaxies depends on their properties, such as morphology (e.g., Hubble 1936; Zwicky et al. 1968; Davis \& Geller 1976; Dressler 1980; Postman \& Geller 1984; Guzzo et al. 1997; Willmer, da Costa \& Pellegrini 1998; Zehavi et al. 2002; Goto et al. 2003), luminosity (e.g., Davis et al. 1988; Hamilton 1988; White, Tully, \& Davis 1988; Park et al. 1994; Lovedav et al. 1995; Guzzo et al. 1997; $\quad$ Benoist et al. 1996; Norberg et al. 2001: Zehavi et al. 2002, 2005b; Coil et al. 2006, 2008), color (e.g., Willmer, da Costa \& Pellegrini 1998; Brown. Webster \& Bovle 2000; Zehavi et al. 2002, 2005b; Coil et al. 2008), and spectral type (e.g., Norberg et al. 2002; Budavari et al. 2003; Madgwick et al. 2003). Galaxy clustering thus provides important clues to the physics of galaxy formation. Often found to reside in galaxy groups and clusters, luminous red galaxies (LRGs) constitute the bright end of the galaxy luminosity function. Clustering of LRGs encodes information about their environments, which

\footnotetext{
1 Institute for Advanced Study, Einstein Drive, Princeton, NJ 08540; zhengz@ias.edu

2 Hubble Fellow

3 John Bahcall Fellow

4 Department of Astronomy, Case Western Reserve University, 10900 Euclid Avenue, Cleveland, OH 44106; izehavi@astronomy.case.edu

5 Steward Observatory, University of Arizona, 933 N. Cherry Avenue, Tucson, AZ 85121; deisenstein@as.arizona.edu

6 Department of Astronomy and Center for Cosmology and AstroParticle Physics, Ohio State University, 140 W. 18th Avenue, Columbus, OH 43210; dhw@astronomy.ohio-state.edu

7 Shanghai Astronomical Observatory, Joint Institute for Galaxy and Cosmology (JOINGC) of SHAO and USTC, Nandan Road 80, Shanghai, 200030, China; ypjing@shao.ac.cn
}

are typically the central regions of groups and clusters. The LRG redshift sample (Eisenstein et al. 2001) of the Sloan Digital Sky Survey (SDSS; York et al. 2000) provides an enormous data set with which to measure the clustering of LRGs. In this paper, we perform theoretical modeling of auto-clustering of LRGs and cross-clustering of LRGs with other types of galaxies to understand the origin of their clustering properties, to learn how they are distributed among massive dark matter halos, and to aid the study of formation and evolution of massive galaxies.

The theoretical understanding of galaxy clustering has been greatly enhanced through the framework of the halo occupation distribution (HOD, see, e.g., Jing. Mo, \& Börner 1998; Ma \& Frv 2000; Peacock \& Smith 2000; Seliak 2000; Scoccimarro et al. 2001; Berlind \& Weinberg 2002) and the closely related approach of the conditional luminosity function (CLF, Yang, Mo, \& van den Bosch 2003). The HOD formalism describes the bias relation between galaxies and matter at the level of individual virialized dark matter halos, whose distribution and properties can be readily predicted by numerical simulations or analytic models given a cosmological model. The key ingredients of this formalism are the probability distribution $P(N \mid M)$ that a halo of mass $M$ contains $N$ galaxies of a given type and spatial and velocity distributions of galaxies within halos. In the CLF approach, the dependence of $P(N \mid M)$ on galaxy luminosity is implicitly derived by inferring the conditional luminosity distribution of galaxies as a function of halo mass $M$. Given the HOD/CLF and the cosmology, any statistics of galaxy clustering on any scales can be predicted, therefore the HOD/CLF method provides a complete description of 
the bias relation between galaxies and dark matter. HOD/CLF modeling has been applied to interpret galaxy clustering measurements in several galaxy surveys (e.g., Jing \& Börner 1998; Jing. Börner, \& Suto 2002; $\quad$ Bullock. Wechsler, \& Somerville 2002; Moustakas \& Somerville 2002; van den Bosch et al. 2003; $\quad$ Magliocchetti \& Porciani 2003; Yan, Madgwick, \& White 2003; Zheng 2004; Yang et al. 2005; Zehavi et al.|2005b; Lee et al.|2006; Hamana et al. 2006; Cooray 2006; Conroy et al. 2006; White et al. 2007; Zheng. Coil. \& Zehavi 2007; Blake et al. 2008; Wake et al. 2008). HOD/CLF analysis recasts galaxy clustering measurements into a form that is more physically informative and conducive for testing galaxy formation theories (see, e.g., Berlind \& Weinberg 2002; Berlind et al. 2003; van den Bosch et al. 2003; Zheng et al. 2005). Zehavi et al. (2005b) present clustering measurements and HOD modeling for galaxies in the SDSS main galaxy spectroscopic sample (Strauss et al. 2002). The HOD modeling results of the luminosity and color dependence of galaxy clustering are found to be in good qualitative agreement with predictions from galaxy formation models (Zheng et al. 2005).

In this paper, we apply HOD modeling to interpret clustering in the SDSS LRG spectroscopic sample, which uses color and magnitude cuts to effectively select LRGs out to redshift $z \sim 0.45$ (Eisenstein et al. 2001). The large volume probed by the LRG sample has led to the detection of the baryon acoustic peak in the two-point correlation function (Eisenstein et al. 2005b). Zehavi et al. (2005a) report the measurements of twopoint correlation functions of 35,000 LRGs on scales of $0.3-40 h^{-1} \mathrm{Mpc}$. They find that LRGs are highly clustered (correlation length $\sim 10 h^{-1} \mathrm{Mpc}$ ) and that more luminous LRGs are more clustered. Clear deviations from a power law are seen in the correlation functions, with a dip at $\sim 2 h^{-1} \mathrm{Mpc}$. Eisenstein et al. (2005a) measure the two-point cross-correlation between 32,000 spectroscopic LRGs and 16 million galaxies in the SDSS imaging sample. Since these reference galaxies have luminosities around $L_{*}$, the characteristic luminosity of the Schechter (1976) luminosity function, they are denoted as $L_{*}$ galaxies. Eisenstein et al. (2005a) find a strong luminosity dependence of the LRG- $L_{*}$ cross-clustering amplitude. The form of the luminosity dependence is itself dependent on scale, with more variation in the clustering amplitude on small scales. Understanding all these autoand cross-clustering features is one of the goals of our HOD modeling. Since LRGs trace massive halos, the cross-correlation between LRGs and $L_{*}$ galaxies also allows us to study the HOD of $L_{*}$ galaxies in these massive halos.

The structure of this paper is as follows. In $\S 2$, we briefly describe the SDSS LRG and $L_{*}$ galaxy samples we use and our modeling method. In $\S 3$, we describe our HOD parameterization for LRG samples and $L_{*}$ samples. In $\S 4$, we perform HOD modeling for LRG twopoint auto-correlation functions and the two-point crosscorrelation functions between LRGs and $L_{*}$ galaxies. We show how LRGs occupy dark matter halos and how $L_{*}$ galaxies occupy massive halos. Based on the modeling results, we interpret the luminosity dependence of the cross-clustering. Finally, we summarize and discuss our results in $\S 5$. We also include three appendices in the paper. In Appendix A, we investigate which HOD parameter plays the major role in the departures of the galaxy two-point correlation function from a pure power law. In Appendix B we present HOD modeling results for two luminosity threshold LRG samples for different cosmological models. In Appendix C, we have a brief discussion on the mass function of the most massive halos and the fluctuation of the number of massive halos in the volume probed by the SDSS LRG samples.

\section{SAMPLES AND METHOD}

The LRG two-point auto-correlation functions in the SDSS have been measured by Zehavi et al. (2005a) with spectroscopic samples. The luminosity cuts of the two LRG samples at $z \sim 0.3$ we model in this paper are $-23.2<M_{g}<-21.2$ and $-23.2<M_{g}<-21.8$, where $M_{g}$ is the restframe $g$-band absolute magnitude at $z=$ 0.3 computed from the observed $r$-band magnitude with $k$ and passive evolution corrections (Eisenstein et al. 2005a). Since galaxies with $M_{g}<-23.2$ are extremely rare, these can be regarded essentially as luminositythreshold samples. For brevity, we call them the $M_{g}<$ -21.2 sample and the $M_{g}<-21.8$ sample, or simply the faint sample and the bright sample. The comoving number densities of the two samples are $9.73 \times 10^{-5} h^{3} \mathrm{Mpc}^{-3}$ and $2.40 \times 10^{-5} h^{3} \mathrm{Mpc}^{-3}$, respectively. Because of the fiber collision effect, the smallest scale can be probed by the spectroscopic LRG samples is about $0.4 h^{-1} \mathrm{Mpc}$. Masjedi et al. (2006) extend the measurements of the two-point correlation function for the $M_{g}<-21.2$ sample down to a scale of $\sim 0.015 h^{-1} \mathrm{Mpc}$ by cross-correlating the spectroscopic sample with the imaging sample to avoid the fiber collision effect. They find that the realspace two-point correlation function of the $M_{g}<-21.2$ sample roughly follow an $r^{-2}$ profile from $\sim 100 h^{-1} \mathrm{Mpc}$ down to $\sim 0.01 h^{-1} \mathrm{Mpc}$. In this paper, we focus on the measurements from the spectroscopic samples in Zehavi et al. (2005a) and limit ourselves to modeling the clustering on scales above $0.3 h^{-1} \mathrm{Mpc}$.

The two-point cross-correlation functions between spectroscopic LRG samples and the imaging $L_{*}$ galaxy sample in the SDSS at $z \sim 0.3$ are measured by Eisenstein et al. (2005a). The $L_{*}$ galaxy sample we model is the one defined by the luminosity range $M^{*}-0.5$ to $M^{*}+1.0$, which is called the $M^{*}+1.0$ sample in Eisenstein et al. (2005a). For the LRG sample in the cross-clustering, we adopt the luminosity-bin sample defined by $-21.7<M_{g}<-21.2$.

We essentially follow the method presented in Zehavi et al. (2005b) and adopt the improvements of Tinker et al. (2005) for theoretical modeling of the twopoint auto- and cross-correlation functions in the HOD framework. In this paper, more general HOD parameterizations are used, as described in the following sections. While the two-point auto-correlation functions in Zehavi et al. (2005a) are projected along the redshift direction, similar to those in Zehavi et al. (2005b), the $\mathrm{LRG}-L_{*}$ two-point cross-correlation functions $\Delta$ measured in Eisenstein et al. (2005a) are volume-averaged real-space cross-correlation functions $\xi_{\times}$. The average is weighted by a spherical window function,

$$
\Delta(a)=\frac{1}{V} \int_{0}^{\infty} d r 4 \pi r^{2} \xi_{\times}(r) W(r ; a),
$$


where the window function is of the form

$$
W(r ; a)=\frac{r^{2}}{a^{2}} \exp \left(-\frac{r^{2}}{2 a^{2}}\right) .
$$

The effective volume $V$ for the window function is $3\left(2 \pi a^{2}\right)^{3 / 2}$. In our modeling, we perform the same volume average to compute the predicted cross-correlation function.

When performing fits to the two-point auto- and cross-correlation functions, we calculate values of $\chi^{2}$ using the full error covariance matrices, inferred through the jackknife method (see Eisenstein et al. 2005a and Zehavi et al. 2005b for details).

Throughout the paper, we adopt the spatially flat "concordance" cosmological model with the matter density parameter $\Omega_{m}=0.3$ and baryon density parameter $\Omega_{b}=0.047$. We assume adiabatic Gaussian primordial density fluctuations with a power-law index of the spectrum $n_{s}=1$. The r.m.s. matter density fluctuation in spheres of radius $8 h^{-1} \mathrm{Mpc}$ linearly extrapolated to $z=0$ is assumed to be $\sigma_{8}=0.8$. The Hubble constant we use is $h=0.7$ in unit of $100 \mathrm{~km} \mathrm{~s}^{-1} \mathrm{Mpc}^{-1}$. Appendix B presents the dependence of the derived HOD parameters on $\sigma_{8}$ and $\Omega_{m}$ for a five-parameter HOD model. The mean redshift of the above LRG and $L_{*}$ samples is around 0.3 and our model calculations take this into account, i.e., all halo properties are calculated at $z=0.3$ for the adopted cosmology. In general, the comoving unit is adopted for distance, but to be compatible with Eisenstein et al. (2005a), we also use proper units with explicit mention when discussing the crossclustering. Finally, we assume $h=1$ when quoting magnitudes throughout the paper.

\section{HOD PARAMETERIZATION}

\subsection{The Mean Occupation Function of LRGs}

The two LRG samples for which the two-point autocorrelation functions are modeled are nearly luminositythreshold samples. The LRG samples defined in the cross-correlation measurements in Eisenstein et al. (2005a) are luminosity-bin samples. We concentrate on the cross-correlation between $L_{*}$ galaxies and $-21.7<$ $M_{g}<-21.2$ LRGs, together with the two-point autocorrelation functions of the two luminosity-threshold samples with $M_{g}<-21.2$ and $M_{g}<-21.8$. The three LRG samples with different luminosity cuts provide leverage to constrain the relation between galaxy luminosity and halo mass. We therefore first parameterize the LRG HOD in a way that includes the luminosity information.

For HOD parameterization, it has been found to be useful to separate contributions from central and satellite galaxies (Kravtsov et al. 2004; Zheng et al. 2005). Central galaxy luminosity is correlated with the host halo mass. Based on predictions of galaxy formation models (Zheng et al. 2005), we assume that at a fixed halo mass the central galaxy luminosity follows a log-normal distribution and the mean luminosity of this distribution has a power-law form $L_{c}=L_{s}\left(M / M_{s}\right)^{p}$, with $p$ independent of halo mass in the range that LRGs probe. In terms of absolute magnitudes,

$$
M_{g c}=M_{g s}-2.5 p \log \left(M / M_{s}\right),
$$

where $M_{g s}$ is the mean luminosity in halos with a pivot mass $M_{s}$ and is simply set to be -19.8 . We note that in this paper $M$ with a subscript " $g$ " stands for the $g$-band absolute magnitude, while that without the subscript " $g$ " is used for halo mass. With a constant standard deviation $\sigma_{M_{g}}$ in magnitude, the luminosity distribution of central galaxies in halos of mass $M$ is then

$$
\frac{d\left\langle N_{\text {cen }}(M)\right\rangle}{d M_{g}}=\frac{1}{\sqrt{2 \pi} \sigma_{M_{g}}} \exp \left[-\frac{\left(M_{g}-M_{g c}\right)^{2}}{2 \sigma_{M_{g}}^{2}}\right] .
$$

LRG samples are not defined purely through luminosity, since there is a color cut in the LRG selection. Therefore, for an LRG sample with $M_{g}<M_{g \text {,thres, }}$, the mean occupation function of central galaxies is calculated as

$$
\left\langle N_{\mathrm{cen}}(M)\right\rangle=\int_{-\infty}^{M_{g, \text { thres }}} d M_{g} \frac{d\left\langle N_{\mathrm{cen}}(M)\right\rangle}{d M_{g}} f\left(M_{g}\right),
$$

where $f\left(M_{g}\right)$ is the LRG selection function at a given luminosity. Based on the LRG luminosity function and the total galaxy luminosity function, we approximate the selection function as $f\left(M_{g}\right)=-2\left(M_{g}+20.45\right) / 3$ in the range $-21.95<M_{g}<-20.45,0$ for $M_{g}>-20.45$, and 1 for $M_{g}<-21.95$. The HOD of a luminosity-bin galaxy sample is just the difference of those of two luminositythreshold samples.

The theoretically predicted mean occupation function of satellite galaxies for a luminosity-threshold sample is close to a power law (Kravtsov et al. 2004; Zheng et al. 2005). Here, we adopt a more flexible parameterization, which can give us a better idea on the constraining power of the two-point clustering on the HOD. For each luminosity-threshold LRG sample, we parameterize the HOD of satellite LRGs through the mean occupation numbers at five mass scales, and the the mean occupation function is assumed to be a cubic spline curve passing through the five points (see Zheng \& Weinberg 2007). Linear extrapolations are used outside of the ranges of the five points. In addition, we impose the central galaxy cutoff profile $\left\langle N_{\text {cen }}(M)\right\rangle$ to $\left\langle N_{\text {sat }}(M)\right\rangle$. The five mass scales are chosen to be $\log \left(M / h^{-1} M_{\odot}\right)=14.10+0.35 i$ $(i=0,1,2,3,4)$. The mean occupation function of satellites in a luminosity-bin sample is obtained by interpolating the values of $\log \left\langle N_{\text {sat }}(M)\right\rangle$ for the two luminositythreshold samples (linearly in $\log L$ ). The satellite occupation number around the mean is assumed to follow the Poisson distribution (Kravtsov et al. 2004; Zheng et al. 2005).

The parameterization of the halo occupation of LRGs we present here is a hybrid of the CLF framework (Yang. Mo, \& van den Bosch 2003) and the HOD framework (Berlind \& Weinberg 2002) - the distribution of central LRGs is put in a CLF form, while that of the satellite LRGs is in the usual HOD form. This combination allows us to infer information on how luminosity of central galaxies changes with halo mass and at the same time to keep a reasonable number of free parameters.

With the above parameterized HOD, we can easily form the mean occupation functions for the two luminosity-threshold LRG samples and the luminositybin sample given their luminosity cuts. The thirteen free parameters are the pivot mass scale $M_{s}$ and the slope $p$ in the luminosity-mass relation, the scatter $\sigma_{M_{g}}$ of the 
luminosity distribution of central LRGs, and the total of ten spline points of $\left\langle N_{\text {sat }}(M)\right\rangle$ for the two luminositythreshold samples. In Appendix B. we provide HOD modeling results for the two luminosity threshold samples and their cosmology dependence based on a simpler five-parameter HOD model.

\subsection{The Mean Occupation Function of $L_{*}$ Galaxies}

The $L_{*}$ sample in Eisenstein et al. (2005a) is at a mean redshift $z \sim 0.3$. To parameterize the HOD of this $L_{*}$ sample, we make use of the modeling result in Zehavi et al. (2005b) at $z \sim 0$. The $L_{*}$ galaxy sample is a luminosity-bin sample $M^{*}-0.5<M_{r}<M^{*}+1.0$, which can be regarded as the difference between two luminositythreshold samples, $M_{r}<M^{*}+1.0$ and $M_{r}<M^{*}-0.5$. As shown in Kravtsov et al. (2004), at low redshifts, for threshold samples of a fixed (comoving) galaxy number density, the shape of the mean occupation function as a function of redshift approximately remains unchanged. We take the advantage of this presumed property and construct the mean occupation function for $L_{*}$ galaxies at $z \sim 0.3$ based on the HODs of $z \sim 0$ galaxies. We first find out the number densities of the two luminosity-threshold samples with $M_{r}<M^{*}+1.0$ and $M_{r}<M^{*}-0.5$ at $z \sim 0.3$. To do this, we adopt the evolution model of the luminosity function in Blanton et al. (2003) set to $z=0.3$ and obtain the two number densities as $n_{1}=1.1 \times 10^{-2} h^{3} \mathrm{Mpc}^{-3}$ and $n_{2}=1.3 \times 10^{-3} h^{3} \mathrm{Mpc}^{-3}$, respectively. We find the luminosity thresholds $L_{1}$ and $L_{2}$ for $z \sim 0$ galaxies that match the two number densities,

$$
n\left(L>L_{1} ; z \sim 0\right)=n_{1}
$$

and

$$
n\left(L>L_{2} ; z \sim 0\right)=n_{2} .
$$

We then use the results presented in Zehavi et al. (2005b) to infer the HODs at $z \sim 0$ for luminosity-threshold samples with $L_{1}$ and $L_{2}$ as the thresholds. Finally, we take the difference of these two HODs to infer the mean occupation function for galaxies in the $L_{1}<L<L_{2}$ luminosity bin,

$\left\langle N\left(L_{1}<L<L_{2} ; M\right)\right\rangle=\left\langle N\left(L>L_{1} ; M\right)\right\rangle-\left\langle N\left(L>L_{2} ; M\right)\right\rangle$.

We take the shape of this mean occupation function as that for the $L_{*}$ galaxies at $z \sim 0.3$. The form of this mean occupation function is a sum of a square window for central galaxies and a power law with an index 1.10 for satellites. With the shape fixed, we allow the mean occupation function to shift in mass scales. Furthermore, we add freedom to the high mass slope, as described below, because we have better data to constrain it.

As shown later, the small-scale two-point crosscorrelation between $L_{*}$ galaxies and LRGs comes from satellite $L_{*}$ galaxies paired with LRGs. As our goal here is to investigate the $L_{*}$ occupation in high mass halos, it is necessary to introduce additional degrees of freedom in the mean occupation function of the satellite $L_{*}$ galaxies in massive halos. We only adopt the $z \sim 0.3$ mean occupation function for $L_{*}$ satellites, as constructed above, up to $10^{13} h^{-1} M_{\odot}$, and introduce another power law to represent the mean occupation function of (satellite) $L_{*}$ galaxies in halos more massive than
$10^{13} h^{-1} M_{\odot}$. We assume this occupation distribution follows the Poisson distribution with the mean in the form of $N_{t}\left(M / M_{t}\right)^{\alpha_{h}}$. Here $M_{t}$ is a pivot mass scale fixed to be $2.5 \times 10^{14} h^{-1} M_{\odot}, N_{t}$ is the mean occupation number of $L_{*}$ galaxies in halos of this pivot mass, and $\alpha_{h}$ denotes the slope at the high mass end. The scale of the pivot mass is chosen to minimize the correlation between $N_{t}$ and $\alpha_{h}$. In order to have more flexibility, we do not impose continuity at $10^{13} h^{-1} M_{\odot}$.

At first glance, our construction of the $L_{*}$ HOD seems complex, involving theoretical priors and introducing an artificial break in the satellite occupation function. A more general HOD model for $L_{*}$ galaxies could be obtained by parameterizing the HODs of two luminosity threshold samples and taking their difference (e.g., Tinker et al. 2007). However, we only have a small number of data points from the LRG- $L_{*}$ cross-correlation functions to constrain the $L_{*}$ HOD. The constraints on the low mass part of the $L_{*}$ HOD (especially the mean occupation function for central galaxies) come from the the number density of the $L_{*}$ sample and the large scale bias of the cross-correlation. The shape of the low mass part of a general $L_{*}$ HOD can not be well constrained by either the number density or the large scale bias, given that the halo bias factor in this mass range only increases slowly. The constraints to the high mass part of the $L_{*}$ HOD come from the small-scale cross-correlation with the LRGs. Our procedure of the $L_{*}$ HOD parameterization reduces the degrees of freedom in the low mass part of the HOD and allows some flexibility in the high mass part of the HOD which is our focus. Although our parameterization is a restricted one, it is suitable for our purpose of understanding clustering properties in $L_{*}$-LRG cross-correlation. To summarize, we allow the low mass end of the mean occupation function (a square window plus the low-mass power law) to have an overall horizontal shift with the shape fixed. The main role of shifting the low mass part of the $L_{*}$ HOD is to match the number density of the $L_{*}$ sample and the large scale bias of the cross-correlation, while the high mass part of the occupation function of $L_{*}$ galaxies that we are interested in determines the small-scale cross-correlation with the LRGs.

The distribution profile of $L_{*}$ satellite galaxies inside halos is assumed to follow the Navarro-Frenk-White (NFW) profile (Navarro, Frenk, \& White 1995, 1996, 1997) with a concentration parameter $c=c_{0}\left(M / M_{\mathrm{nl}}\right)^{\beta}$, where $M_{\mathrm{nl}} \sim 1.5 \times 10^{12} h^{-1} M_{\odot}$ is the nonlinear mass at $z \sim 0.3$ and $\beta$ is fixed to -0.13 (Bullock et al. 2001). As a whole, there are four free parameters in the $L_{*}$ HOD, the overall horizontal shift of the low mass part, the high mass amplitude $N_{t}$ and slope $\alpha_{h}$ of the mean occupation function, and the halo concentration parameter $c_{0}$ extrapolated to the nonlinear mass scale. To compute the one-halo term of LRG- $L_{*}$ cross-correlation, we make the simplifying assumption that the occupation number of LRGs and that of $L_{*}$ galaxies inside the same halo are uncorrelated. That is, $\left\langle N(\mathrm{LRG} ; M) N\left(L_{*} ; M\right)\right\rangle=$ $\langle N(\mathrm{LRG} ; M)\rangle\left\langle N\left(L_{*} ; M\right)\right\rangle$. We discuss the effect of this assumption on our modeling results in $\S 4$.

\section{MODELING RESULTS}

We model simultaneously the two-point autocorrelation functions of the two luminosity-threshold 

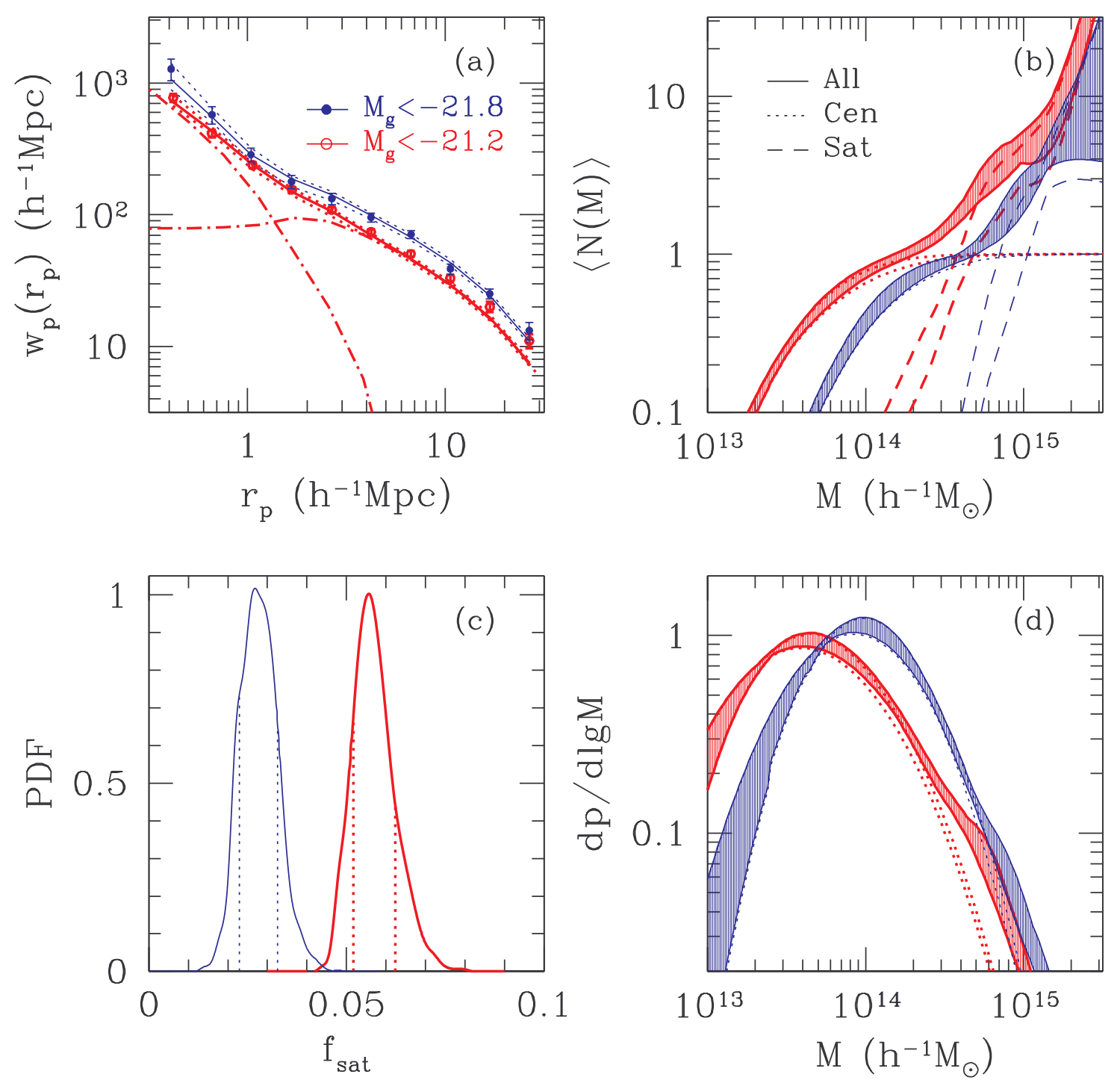

FIG. 1. - Projected two-point auto-correlation functions and best-fit HODs for the two luminosity-threshold LRG samples. Panel (a): The measured two-point correlation functions (data points and error bars) and the HOD model fits (solid curves). The two dashed curves for each sample show the envelope of predictions from models with $\Delta \chi^{2}<4$. The predicted one-halo and two-halo terms (dot-dashed curves) are also shown for the sample with the lower luminosity threshold. Panel (b): The mean occupation functions (solid curves) of LRGs from the best fits, with contributions from central (dotted) and satellite (dashed) LRGs. For each sample, the two sets of curves are the envelope from models with $\Delta \chi^{2}<4$ and those for all galaxies are shaded. Panel $(c)$ : The marginalized distribution of the satellite fraction in each sample with the central $68 \%$ distribution marked by the two dashed lines. Panel $(d)$ : The probability distribution of halo masses for the LRGs in each sample (solid lines), obtained from the occupation function shown in panel $(b)$ weighted by the differential halo mass function. The dotted lines show the halo mass probability distribution from just the central galaxies.

LRG samples $\left(M_{g}<-21.2\right.$ and $\left.M_{g}<-21.8\right)$ and the two-point cross-correlation function between the $L_{*}$ and the luminosity-bin $\left(-21.7<M_{g}<-21.2\right)$ LRG sample. Altogether, there are seventeen free parameters, four for the $L_{*}$ HOD and thirteen for the LRG HOD.

In addition to the sum of $\chi^{2} \mathrm{~s}$ from the two autocorrelation functions and the cross-correlation function, we also add the number densities of the two threshold LRG samples and the $L_{*}$ galaxies into the overall $\chi^{2}$. That is,

$$
\begin{aligned}
\chi^{2} & =\left(\mathbf{w}_{\mathbf{1}}-\mathbf{w}_{\mathbf{1}}^{*}\right){ }^{\mathbf{T}} \mathbf{C}_{\mathbf{1}}^{-\mathbf{1}}\left(\mathbf{w}_{\mathbf{1}}-\mathbf{w}_{\mathbf{1}}^{*}\right) \\
& +\left(\mathbf{w}_{\mathbf{2}}-\mathbf{w}_{\mathbf{2}}^{*}\right)^{\mathbf{T}} \mathbf{C}_{\mathbf{2}}^{-\mathbf{1}}\left(\mathbf{w}_{\mathbf{2}}-\mathbf{w}_{\mathbf{2}}^{*}\right)
\end{aligned}
$$

$$
+\left(\boldsymbol{\Delta}-\boldsymbol{\Delta}^{*}\right)^{\mathbf{T}} \mathbf{C}_{\times}^{-\mathbf{1}}\left(\boldsymbol{\Delta}-\boldsymbol{\Delta}^{*}\right)+\sum_{i=1}^{3} \frac{\left(n_{i}-n_{i}^{*}\right)^{2}}{\sigma_{n_{i}}^{2}}
$$

where $\mathbf{w}_{\mathbf{1}}, \mathbf{w}_{\mathbf{2}}$, and $\boldsymbol{\Delta}$ are the vectors of auto-correlation functions of the two LRG samples and the LRG- $L_{*}$ crosscorrelation function, and $n_{i}(i=1,2,3)$ are the three number densities. The observed values are denoted with a superscript $*$. The full covariance matrix is used for each correlation function and $10 \%$ fractional errors are assumed for each of the galaxy number densities.

The three covariance matrices, $\mathbf{C}_{\mathbf{1}}, \mathbf{C}_{\mathbf{2}}$, and $\mathbf{C}_{\times}$ are estimated using jackknife resampling. For each of the two LRG samples, 104 jackknife subsamples are used (Zehavi et al. 2005a) for estimating the covari- 
ance among 10 data points. For the LRG- $L_{*}$ crosscorrelation, the covariance among 6 data points is estimated with 50 jackknife subsamples (Eisenstein et al. 2005a). Zehavi et al. (2005b) performed extensive tests with mock catalogs to access the reliability of the jackknife error estimates in projected correlation functions over a similar range of separations. They used 100 mock catalogs with the same geometry and angular completeness as the SDSS sample and similar clustering properties, created using the PTHalos method of Scoccimarro \& Sheth (2002). Their tests showed that the jackknife method is a robust way to estimate the error covariance matrix, especially for the relatively large volumes probed here. This holds as long as the number of jackknife realizations, $n$, is significantly larger than the dimension of the data vector, $p$. Hartlap et al. (2007) discusses related issues, pointing out a potential bias in general model fitting which depends on the ratio of $p$ to $n$. As a crude test of our error uncertainties, we incorporate their proposed method to remove this bias by multiplying the inverse of the covariance matrix by a factor of $(n-p-2) /(n-1)$. In our case, this factor is about 0.9 . We perform this for the $M_{g}<21.2 \mathrm{LRG}$ sample, resulting in $a \sim 10 \%$ increase in the uncertainties of the HOD parameters, but no noticeable change in the bestfit values. We note that the results presented later in this paper do not include such a correction.

We assume the different clustering measurements to be independent, ignoring possible correlations between statistical errors across correlation functions and number densities of different samples. Strictly speaking, such statistical correlations are not zero. For example, the $M_{g}<-21.2 \mathrm{LRG}$ sample includes LRGs with $M_{g}<-21.8$, therefore the auto-correlation functions of the $M_{g}<-21.2$ and $M_{g}<-21.8$ samples are partially correlated. These correlations could be estimated with the jackknife technique, but they would be noisy. Neglecting such correlations might make the constraints on the HOD parameters somewhat tighter than they should be, which is a caveat for interpreting our results.

We adopt a Markov Chain Monte Carlo technique (MCMC; e.g., Gilks, Richardson, \& Spiegelhalter 1996) to explore the HOD parameter space. At each point of the chain, we take a random walk in the parameter space to generate a new set of HOD parameters. The step-size of the random walk for each parameter is drawn from a Gaussian distribution. The probability to accept the new set of HOD parameters depends on the difference between $\chi_{\text {old }}^{2}$ and $\chi_{\text {new }}^{2}$ (the values of $\chi^{2}$ for the new and old models): 1 for $\chi_{\text {new }}^{2} \leq \chi_{\text {old }}^{2}$ and $\exp \left[-\left(\chi_{\text {new }}^{2}-\chi_{\text {old }}^{2}\right)\right]$ for $\chi_{\text {new }}^{2}>\chi_{\text {old }}^{2}$. Flat priors in logarithmic space are adopted for the LRG satellite occupation numbers at the ten spline points and the pivot mass scale $M_{s}$ and for $N_{t}$ and mass scale shift of $L_{*}$ galaxies. Flat priors in linear space are used for other HOD parameters.

The total number of data points to model is $29(10+10$ auto-correlation measurements for the two LRG samples, 6 LRG- $L_{*}$ cross-correlation measurements, and 3 number densities). The number densities are well reproduced by our model, with the median values $1.1-\sigma, 0.4-\sigma$, and 0.1- $\sigma$ away from the observed ones for the two LRG and the $L_{*}$ samples. Our model has 17 free parameters and therefore the number of degree of freedom is 12 . We find that the best-fit model has $\chi^{2}=25$. The probability for a $\chi^{2}$ value higher than our best-fit $\chi^{2}$ is $1.5 \%$. (Including the Hartlap et al. (2007) correction gives $\chi^{2} \sim 22$ and the probability increases to $\sim 4 \%$ ). The relatively-large $\chi^{2}$ values might be partly caused by our neglecting the correlation between statistical errors across correlation functions and number densities of different samples. It also indicates that the accuracy in our analytical model of the two-point galaxy correlation function needs to be improved and that our HOD parameterization is not perfect. With the above caveats in mind, we present our modeling results below.

\subsection{Constraints on the HOD of LRGs}

\subsubsection{HOD for the Luminosity-threshold LRG Samples}

Figure 1] shows the fitting results for the two luminosity-threshold LRG samples. Figure $11 a$ shows the best-fit projected two-point correlation functions together with the measurements. For the $M_{g}<-21.2$ sample, the one-halo and two-halo components (dot-dashed curves) of the fit are also shown. In Figure $1 b$, the mean occupation function for each sample is plotted, separating into contributions from central (dotted) and satellite (dashed) galaxies. The range of mean occupation functions with $\Delta \chi^{2}<4$ is denoted by the shaded region $\left(\Delta \chi^{2}<4\right.$ is chosen so that the envelopes are sampled by a large number of MCMC points and the range delineated by the envelopes can be clearly seen in the plot). As in Zehavi et al. (2005b), the amplitude of the high halo mass end of the mean occupation function is poorly constrained by two-point correlation functions. Since the abundance of halos drops exponentially at the high mass end, the two-halo pairs are mostly contributed by lower mass halos. Although the number of one-halo pairs per halo rises roughly as $\langle N(M)\rangle^{2}$, the exponential drop of the number of high mass halos also makes most one-halo pairs come from lower mass halos. Overall, the two-point correlation function is dominated by signals from halos of lower mass where $\langle N(M)\rangle \sim$ a few, leading to the poor constraints on the HOD at the very high mass end. The constraint on the high mass end slope of $\left\langle N_{\text {sat }}(M)\right\rangle$ for the faint LRG sample appears to be relatively strong, and we discuss its possible implications in $\S 5$.

The results show that the host halos of LRGs are massive, above $10^{13} h^{-1} M_{\odot}$, corresponding to large galaxy groups and clusters, which is consistent with studies of the environment of luminous galaxies (e.g., Loh 2003). We find that as the threshold luminosity increases by a factor of $\sim 1.7$, the mass scale of host halos shifts by a factor of $\sim 2.3$, which implies a correlation between the luminosity $L_{c}$ of the central LRG and the mass $M$ of the host halo in the form of $L_{c} \propto M^{0.66}$.

LRGs are often thought to be the central elliptical galaxies of galaxy groups of clusters. Our results clearly show that a fraction of the LRGs have to be satellite galaxies. The main constraint for this comes from the high amplitudes of the correlation function at small scales. Without satellite LRGs, the projected correlation function would flatten out toward small scales, similar in shape to the two-halo term shown in Figure 1 $1 a$. To explain the rising small-scale clustering, the one-halo term has to be introduced, hence we require the existence of satellite LRGs. However, the fits imply that 

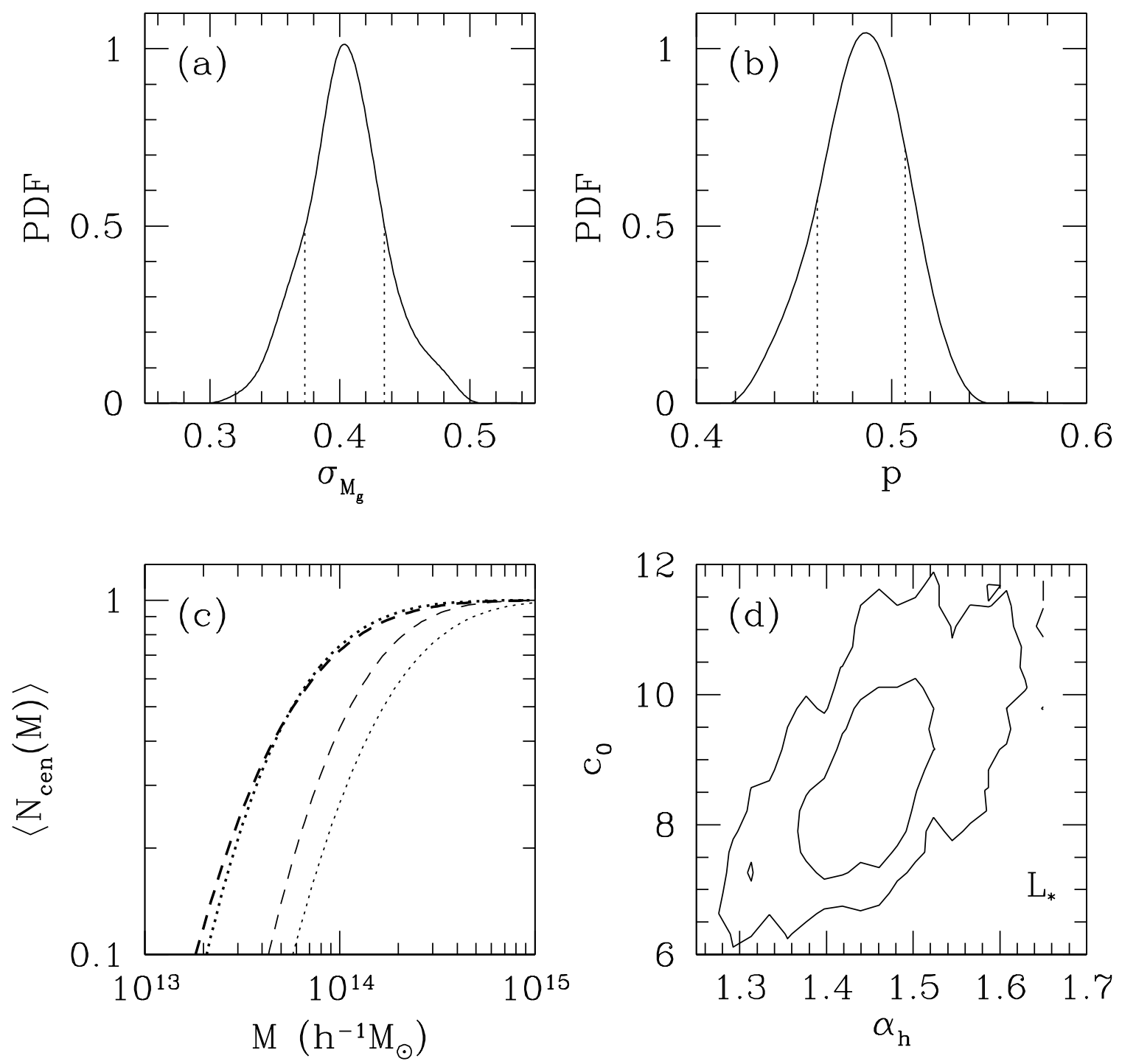

FIG. 2.- Constraints on HOD parameters from the LRG auto-correlation functions and the LRG- $L_{*}$ cross-correlation function (see the text for details). Panel (a): The marginalized distribution of the parameter $\sigma_{M_{g}}$, which is the width (in magnitude) of central galaxy luminosity distribution at a fixed halo mass. Panel $(b)$ : The marginalized distribution of the parameter $p$, which characterizes the relation between mean central galaxy luminosity and halo mass through $L_{c} \propto M^{p}$. In panels $(a)$ and $(b)$, the two dashed vertical lines indicate the central $68 \%$ of the distribution. Panel $(c)$ : Illustration of the effect of $\sigma_{M_{g}}$ and $p$ on the mean occupation functions of LRGs. The thick dotted and dashed curves are for the $M_{g}<-21.2$ LRG sample, with $\sigma_{M_{g}}$ varied from 0.37 to 0.43 (i.e., a $\pm 1 \sigma$ change). The thin dashed and dotted curves are for the $M_{g}<-21.8$ LRG sample, with $p$ varied from 0.46 to $0.51(\mathrm{a} \pm 1 \sigma$ change in $p$ ). Panel $(d)$ : The marginalized joint distribution of the concentration parameter $c_{0}$ (normalized to that at the $z \sim 0.3$ nonlinear mass scale) and the high mass slope $\alpha_{h}$ of the mean occupation function for the $L_{*}$ galaxies. The contours show the $68 \%$ and $95 \%$ confidence levels for two parameters.

only a small fraction of LRGs are satellites: $5.2-6.2 \%$ and $2.3-3.2 \%$ in the $M_{g}<-21.2$ and $M_{g}<-21.8$ samples, respectively, as shown in Figure 1c. The steeper rise of the small scale clustering makes the two-point correlation function of the brighter LRG sample deviate from a power law more prominently. In Appendix A we investigate the key ingredients that lead to the departure from a power law and provide insight as to why the departure becomes more clear for samples that are more luminous (as shown here) and for samples at higher redshifts (e.g., Ouchi et al. 2005).

The mean occupation function tells us the mean number of LRGs as a function of the halo mass. The result can be viewed differently by asking what the probability distribution of masses of halos hosting such LRGs would be. The probability is simply the product of the mean occupation function and the differential halo mass function. We show such probability distributions from the MCMC run in Figure 11. The distribution of $M_{g}<-21.2$ LRGs peaks at $\sim 4.5 \times 10^{13} h^{-1} M_{\odot}$, and for $M_{g}<-21.8 \mathrm{LRGs}$ the peak shifts to higher mass, $\sim 10^{14} h^{-1} M_{\odot}$. Both distributions span a large range in halo mass. The full width at half maximum (FWHM) for either distribution is about $\Delta \log M=0.8$. Since only a small fraction of LRGs are satellites, the probability distribution is almost determined by the central LRGs. Only in halos more massive than a few times $10^{14} h^{-1} M_{\odot}$ does the 
number of satellites in a halo become significant (i.e., greater than one on average), and around this mass scale a low amplitude shoulder in the distribution emerges. In FWHM sense, we find that $M_{g}<-21.2$ and $M_{g}<-21.8$ LRGs reside in halos of mass $2-13 \times 10^{13} h^{-1} M_{\odot}$ and $4-$ $25 \times 10^{13} h^{-1} M_{\odot}$, respectively.

From the mean occupation functions in Figure $1 b$, one also notices that, compared with the samples of lower luminosities in Zehavi et al. (2005b), the low mass cutoff profiles for the LRG samples are better constrained. The relatively tight constraints come from the steepening both in the halo mass function and in the mass dependence of the halo bias factor toward high halo mass. The former steepening makes the galaxy number density more sensitive to the cutoff profile, while the latter one increases the sensitivity of the large-scale galaxy bias factor to the cutoff profile. With our HOD parameterization, the cutoff profile of the LRG mean occupation function encodes information on the distribution of central galaxy luminosity in halos of fixed mass and on how the mean central galaxy luminosity scales with halo mass. Figure $2 a$ shows the marginalized distribution of the width $\sigma_{M_{g}}$ (in magnitude) of the (log-normal) central galaxy luminosity distribution at a fixed halo mass. The clustering data require a scatter of $\sim 0.16$ dex in the central galaxy luminosity in halos of a given mass. The thick dotted and dashed curves in Figure $2 c$ shows how $\sigma_{M_{g}}$ affects the cutoff profile in $\left\langle N_{\text {cen }}(M)\right\rangle$ of the $M_{g}<-21.2$ sample by varying $\sigma_{M_{g}}$ by $\pm 1 \sigma$. We note that the constraints on the cutoff profile (and therefore $\sigma_{M_{g}}$ ) depend on cosmological parameters, especially $\sigma_{8}$, in the sense of a larger $\sigma_{M_{g}}$ for a larger $\sigma_{8}$ (see Appendix B for more details).

The mean central galaxy luminosity scales with halo mass, which is characterized by the parameter $p, L_{c} \propto$ $M^{p}$. LRG samples with different luminosity cuts allow us to constrain the parameter $p$. The marginalized distribution of $p$ is shown in Figure $2 b$. The thin dotted and dashed curves in Figure 2 $c$ show the effect $p$ on the mass scale shift of the $M_{g}<-21.8$ sample relative to the $M_{g}<-21.2$ sample. The value of $p$ is around 0.48 , which seems to be inconsistent with the value 0.66 estimated from comparing the luminosity and halo mass scale of the two threshold LRG samples. The reason is simple - in our parameterization, the parameters $p$ and $\sigma_{M_{g}}$ constrained here correspond to all central galaxies, not only central LRG galaxies that have a color selection criterion imposed.

Our HOD parameterization here is rather flexible in the satellite HOD. Again, in Appendix B, we present modeling results with a five-parameter HOD model and show their dependence on cosmology. In $\S$, we compare our HOD modeling results with those from other works and discuss a few issues related to the modeling.

We note that, on large scales, the bestfit $w_{p}$ curve in Figure $19 a$ appears to be lower than that from the fiveparameter model (Fig. 8 3 a) for the $M_{g}<-21.2$ sample. The low mass cutoff profiles of $\left\langle N_{\text {cen }}(M)\right\rangle$ for the bright and the faint LRG samples are correlated in the parameterization adopted here, which means that the HOD for central galaxies is more restrictive than that in the five-parameter model. The LRG- $L_{*}$ cross-correlation also limits the range of LRG HODs. The bestfit solution is a compromise in matching the large-scale amplitudes of both the LRG auto-correlation functions and the LRG$L_{*}$ cross-correlation function. The result implies that our HOD parameterization is not perfect and that there is room to improve it (e.g., by allowing the scatter in the central galaxy luminosity to vary with halo mass).

\subsubsection{Mass Scales of Host Halos of Central and Satellite LRGs}

Applying HOD modeling to galaxy samples with different luminosity thresholds, Zehavi et al. (2005b) find that there is a remarkable scaling relation between the characteristic minimum mass $M_{\min }$ of the host halos and the mass scale $M_{1}$ of halos that on average host one satellite galaxy (above the luminosity threshold) in addition to the central galaxy, $M_{1} \sim 23 M_{\text {min }}$. With a HOD parameterization close to what is used here, Zheng, Coil, \& Zehavi (2007) found the relation is more like $M_{1} \sim 18 M_{\min }$. Given the parameterization described in 3.1 we define $M_{\min }$ to be the halo mass at which the expected number of central galaxies above the luminosity threshold is 0.5 , whether or not the galaxy satisfies the LRG color criteria. Theoretical studies of HODs of subhalos in dissipationless dark matter simulations (Kravtsov et al. 2004) and those predicted by SPH and semi-analytic galaxy formation models (Zheng et al. 2005) reveal a similar relation with the scaling factor around 20. As shown by Berlind et al. (2003), the large gap between $M_{1}$ and $M_{\text {min }}$ arises because in the low occupation regime, a more massive halo tends to host a more massive central galaxy, rather than multiple smaller galaxies. Does the $M_{1}-M_{\min }$ scaling relation extend to massive halos hosting LRGs?

In Figure 3, we plot $M_{\min }$ and $M_{1}$ as a function of the threshold luminosity from Zheng, Coil, \& Zehavi (2007), corrected to be consistent with $\sigma_{8}=0.8$ adopted in this paper. The luminosity in Zheng. Coil, \& Zehavi (2007) is in $z=0.1 r$-band (see Zehavi et al. 2005b). For comparison, we convert the $K$-corrected and passively evolved $z=0.3 \mathrm{~g}$-band threshold luminosities of the two LRG samples to the $z=0.1 r$-band ones by adopting an apparent color $g-r=0.4$, and we obtain $M_{r}<-21.6$ and $M_{r}<-22.2$, respectively. We obtain the distribution of $M_{\min }$ and $M_{1}$ for the two luminosity-threshold LRG samples modeled in this paper by solving $\left\langle N_{\text {cen }}(M)\right\rangle=0.5$ and $\left\langle N_{\text {sat }}(M)\right\rangle=1$ for each set of HOD parameters in the MCMC chain. The results are shown as the last two pairs of points. Roughly speaking, they seem to follow the previous trend. The mild discontinuity probably reflects an imperfect magnitude conversion, which is not surprising as we are trying to account for filter difference, $K$-correction, and stellar population evolution from $z \sim 0.3$ to $z \sim 0.15$. A more interesting difference is that the scaling factors between $M_{1}$ and $M_{\text {min }}$ become $10.5_{-1.5}^{+5.1}\left(M_{g}<-21.2\right)$ and $7.2_{-0.8}^{+0.9}\left(M_{g}<-21.8\right)$, respectively. For comparison, the mean $M_{1}$-to- $M_{\min }$ ratio for the MAIN galaxy samples shown in Figure 3 is 18.3 with a mean $1 \sigma$ uncertainty of $\sim 3.6$. This indicates that the scaling relation may break down in very massive halos. In fact, the scaling relation from the MAIN sample with the highest luminosity threshold $\left(M_{r}<-21.5\right)$ in Figure 3 has already shown a trend of such a decrease, with an $M_{1}$-to- $M_{\min }$ ratio of $13.2_{-2.4}^{+2.7}$. As a whole, these results show that the $M_{1}-M_{\text {min }}$ scaling factor decreases 


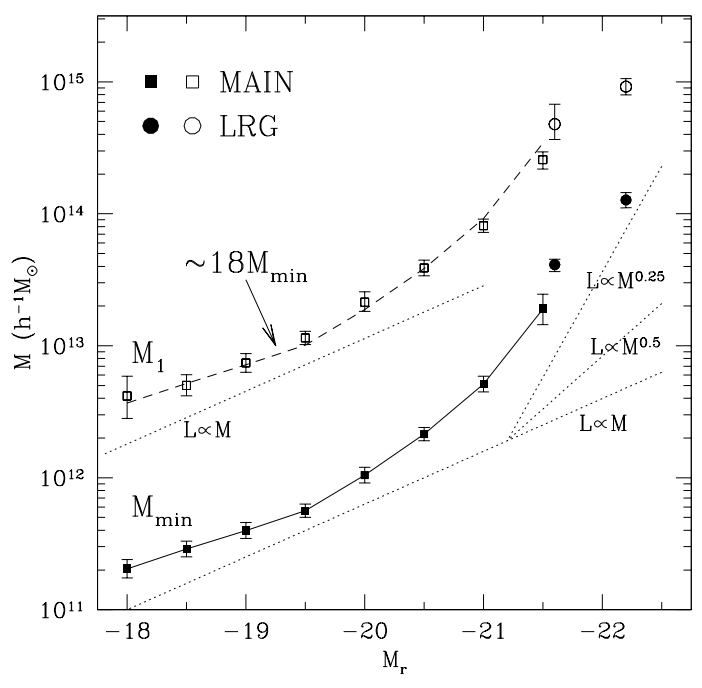

FIG. 3.- Mass scales of the LRG HODs as a function of threshold luminosity. Shown are the relation between the characteristic minimum mass $M_{\min }$ at which $50 \%$ of halos host central galaxies above the luminosity threshold and the mass $M_{1}$ of halos that on average host one satellite galaxy, as a function of the threshold absolute magnitude. The square points are taken from Zheng. Coil, \& Zehavi (2007) for the SDSS main galaxy sample (corrected to $\sigma_{8}=0.8$ ). Open and filled circles are the results for the two LRG samples (note that the $z=0.3 \mathrm{~g}$-band luminosity is converted to $z=0.1 r$-band by adopting an apparent color of $g-r=0.4$ ). Dotted lines indicate different relations between luminosity of central galaxy and mass of host halo.

for luminous galaxies that reside in massive halos. We caution, however, that the definition of the $M_{1} / M_{\text {min }}$ ratio becomes more parameterization dependent at high galaxy luminosities because the scatter between luminosity and halo mass is larger (i.e., the low mass cutoff of the HOD is less sharp).

The change of the $M_{1}-M_{\min }$ scaling factor in massive halos can be understood from the competition between accretion and destruction processes. In general, massive halos accrete their satellites more recently than less massive halos. While the rate of satellite accretion for low mass halos peaks at $\sim 10 \mathrm{Gyr}$ ago, cluster-sized halos constantly accrete satellites until recently (Zentner et al. 2005). As a consequence, there is less time for the orbit of satellites in a massive halo to decay through dynamical friction and for them to merge with the central galaxy to form a larger (brighter) central galaxy. In addition, the LRG samples we study are at redshift $\sim 0.3$, which makes the accretion even more dominant. Therefore, the decrease in the ratio of $M_{1}$ and $M_{\text {min }}$ could be a manifestation of the favor of accretion over destruction in massive halos.

\subsubsection{HOD for the Luminosity-Bin LRG Sample}

The cross-correlation between luminosity-bin LRG sample $\left(-21.7<M_{g}<-21.2\right)$ and the $L_{*}$ galaxies also leads to constraints on the HOD of the luminosity-bin LRG sample. The lower (solid) curves in the right panel of Figure 4 are the $\Delta \chi^{2}<4$ envelope of the mean occupation function of the $-21.7<M_{g}<-21.2$ LRGs. The bump (dotted) around $\sim 10^{14} h^{-1} M_{\odot}$ is the contribution from central galaxies and the dashed curves represent contributions from satellites. The result shows that most of the LRGs in this luminosity bin are central galaxies in halos of mass $\sim 2-20 \times 10^{13} h^{-1} M_{\odot}$, and a small fraction $(\sim 7 \%)$ of them are satellites in more massive halos.

\subsection{Constraints on the HOD of $L_{*}$ Galaxies}

Zehavi et al. (2005b) perform HOD modeling of the two-point auto-correlation functions of the MAIN galaxy sample and show that the two-point function can impose important constraints on the HOD of a sample of galaxies. In general, the mean occupation function is tightly constrained around $\langle N(M)\rangle \sim$ a few. It becomes loosely constrained towards higher halo masses because the two-point correlation function is less sensitive to the occupation distribution in these halos as a result of the steep drop of the halo mass function. Therefore, the analyses of the MAIN galaxy sample with low luminosity thresholds in Zehavi et al. (2005b) cannot quite reveal how these low luminosity galaxies reside in massive halos. Since LRGs automatically pick out the massive halos, the cross-correlation between MAIN sample galaxies and LRGs provides us a nice way to study the halo occupation of MAIN sample galaxies in massive halos, enabling us to better constrain the HOD of $L_{*}$ galaxies in massive halos.

Figure $2 d d$ shows constraints on the concentration parameter $c_{0}$ and the high mass slope $\alpha_{h}$ of the mean occupation function for $L_{*}$ galaxies, marginalized over the other parameters. These two parameters are correlated in a sense that a higher $c_{0}$ corresponds to a higher $\alpha_{h}$. Higher $\alpha_{h}$ means that more $L_{*}$ galaxies reside in higher mass halos with lower concentrations and larger virial radius, so to maintain the small-scale (cross-)clustering the distribution of galaxies need to be more concentrated, i.e., a higher $c_{0}$. The high mass slope of the mean occupation function of $L_{*}$ galaxies is $1.49 \pm 0.09$ (1- $\sigma$ range), steeper than the value 1.10 at lower mass. The concentration parameter $c_{0}$ represents the value extrapolated to $M_{\mathrm{nl}} \sim 1.5 \times 10^{12} h^{-1} M_{\odot}$ according to $c=c_{0}\left(M / M_{\mathrm{nl}}\right)^{-0.13}$. Its 1- $\sigma$ range is found to be $9.4 \pm 1.7$, which translates to $5.4 \pm 1.0$ and $4.0 \pm 0.7$ in halos of $10^{14} h^{-1} M_{\odot}$ and $10^{15} h^{-1} M_{\odot}$, respectively, implying that the distribution of $L_{*}$ galaxies more or less follows that of the dark matter, at radii the data can probe ( $\gtrsim 0.2 h^{-1} \mathrm{Mpc}$ comoving). The mean occupation function of $L_{*}$ galaxies in massive halos is shown as upper (solid) curves in the right panel of Figure 4. These two curves are the envelope determined by $\Delta \chi^{2}<4$. On average, about ten $L_{*}$ galaxies are expected to reside in a halo of mass $\sim 10^{14} h^{-1} M_{\odot}$.

Our fits show that the mean occupation function of $L_{*}$ galaxies at high halo masses becomes steeper than the slope 1.10 at intermediate masses (and lower redshift). We have also run a model with fixed slope 1.10 for the satellite mean occupation function in the whole mass range. The fits to the LRG auto-correlation function and the LRG- $L_{*}$ cross-correlation function become much worse, with the overall $\chi^{2}$ increasing by $\sim 50$. The resulting best fit to the cross-correlation function is plotted in the left panels of Figure4. The lower panel shows the fractional difference between the fits (thick curve for the flexible model and thin curve for the one with fixed slope) and the data. The fit from the model with fixed slope is almost on top of the one from the more flexible model, but the error bars in the measurement are small 

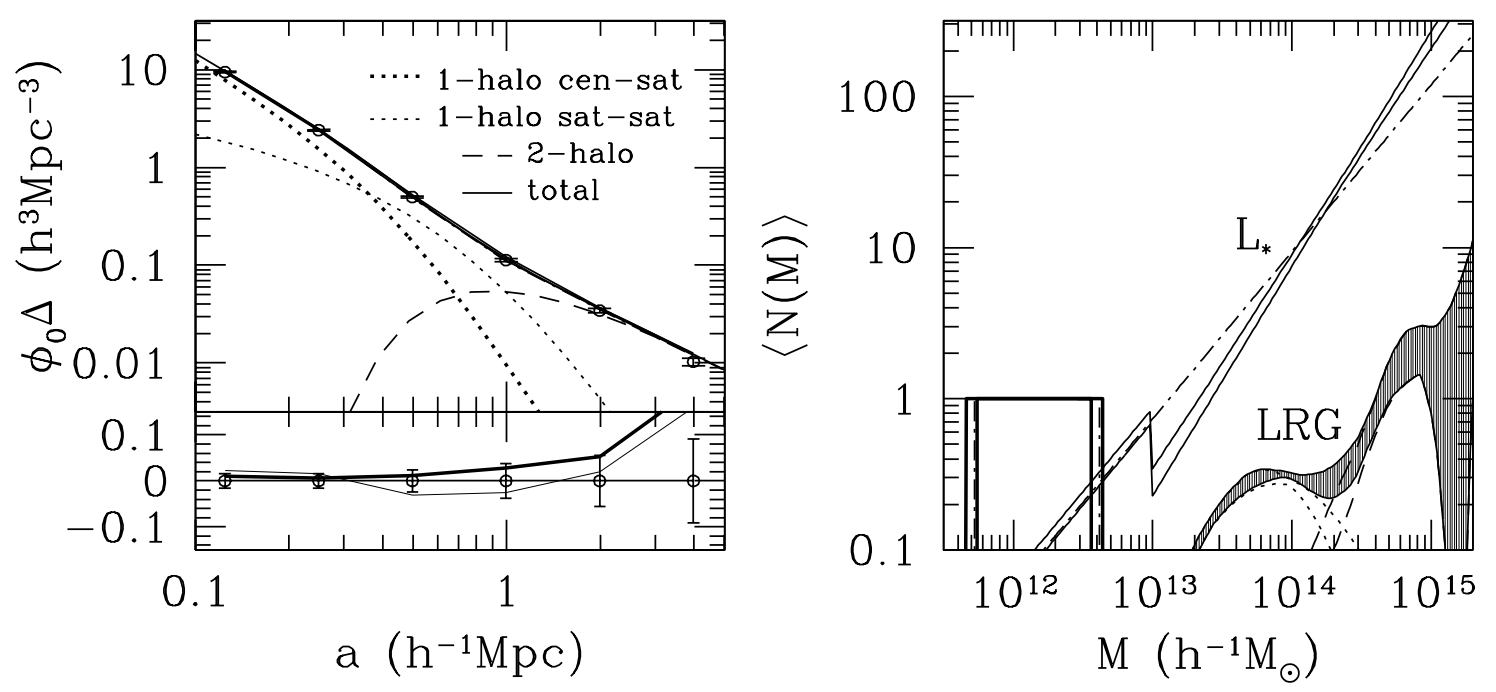

FiG. 4.- Mean occupation functions and cross-correlation functions of $L_{*}$ galaxies and LRGs from the HOD modeling. Left panels: the predicted two-point cross-correlation function between LRGs and $L_{*}$ galaxies separated into contributions from central and satellite LRGs paired with $L_{*}$ galaxies within same halos and LRGs paired with $L_{*}$ galaxies from different halos. The scale $a$ is in units of proper $h^{-1}$ Mpc to be consistent with that adopted in Eisenstein et al. (2005a). The quantity $\phi_{0}=2.267 \times 10^{-2} h^{3} \mathrm{Mpc}^{-3}$ is the proper number density of $L_{*}$ galaxies at the mean redshift $z \sim 0.3$. The lower panel shows the fractional difference between model fits (thick for the flexible model and thin for the slope-fixed model) and data (see the text). Right panel: the mean occupation function for the $L_{*}$ galaxies in massive halos (top solid curves) and that for the luminosity-bin LRGs. (bottom curves). The square window at the low mass end represents the mean occupation function of central $L_{*}$ galaxies. The envelopes of the mean occupation functions are derived from models with $\Delta \chi^{2}<4$. Dotted and dashed curves in the LRG mean occupation function are contributions from central and satellite galaxies, respectively. The dot-dashed curve shows the mean occupation function for the $L_{*}$ galaxies from a model with the slope fixed to be 1.10 in the whole mass range.

and the change in $\chi^{2}$ is substantial (an increase of $\sim 10$ in the $\chi^{2}$ contributed by the cross-correlation function). The corresponding high-mass end of the mean occupation function of $L_{*}$ galaxies from this more restricted model is shown as the dot-dashed line in the right panel of Figure 4. The single-slope model has more $L_{*}$ satellites in halos less massive than $10^{14} h^{-1} M_{\odot}$, and the best-fit concentration parameter for $L_{*}$ galaxies is about a factor of two smaller; these two effects compensate each other to approximately maintain the amplitude of the small-scale cross-correlation. Unless the error bars in the measurements were underestimated (e.g., by a factor of two), the restricted model is highly disfavored by its much worse fits to the data $\left(\Delta \chi^{2} \sim 50\right)$.

The cause and implication of the steep inferred high mass slope of the $L_{*}$ galaxy occupation function is not clear. It may be related to the selection of $L_{*}$ galaxies - the sample used here is composed of galaxies in a bin of 1.5 magnitude around a redshift-dependent reference magnitude that is supposed to match $L_{*}$ at $z \sim 0.3$ (Eisenstein et al. 2005a). Since the redshift range $(0.16<z<0.44)$ is not narrow, the $L_{*}$ sample should be regarded as an effective sample, rather than a uniform sample. It may also be caused by the imperfection in the analytic model of the two-point crosscorrelation function. The small measurement errors in the two-point cross-correlation function may require a more accurate model than the one used in this paper, and allowing for uncertainty in the model accuracy would increase the allowed range of the high mass slope. Finally, it may also be related to the assumption that the occupation numbers of LRGs and $L_{*}$ galaxies in the same halo are uncorrelated ( $\S 3.2$ ). Since the effect of any correlation between the two occupation numbers be- comes smaller as halo mass increases (Simon et al. 2009), an anti-correlation between the LRG and $L_{*}$ occupation numbers would lead to a decrease in the high mass slope of the $L_{*}$ galaxy to conserve the number of LRG- $L_{*}$ pairs. As a whole, we therefore have higher confidence in the value of $\langle N\rangle \sim 10$ at $M \sim 10^{14} h^{-1} M_{\odot}$, where the fits from the two models cross, than we have in the slope $\alpha_{h}$ itself.

The modeling result also shows (see the left panel of Figure 4) that the LRG- $L_{*}$ cross-correlation function is dominated by central LRGs paired with satellite $L_{*}$ galaxies on scales less than $\sim 0.5 h^{-1} \mathrm{Mpc}$ (comoving), while above this scale the signal from satellite LRGs paired with satellite $L_{*}$ galaxies takes over until $\sim 1.5 h^{-1} \mathrm{Mpc}$ (comoving), where the two-halo pairs start to dominate. We show below that the variation with scales in the contributing components is the key to understanding the scale dependence of the luminositydependent cross-clustering. In the one-halo term, the signal from central LRGs mainly comes from halos of mass a few times $10^{13}$ to $10^{14} h^{-1} M_{\odot}$, while the signal from satellite LRGs is from halos of $\sim 10^{14.5} h^{-1} M_{\odot}$, where the LRG occupation number is a few. Although the occupation numbers of LRGs and $L_{*}$ galaxies keep rising toward higher halo mass, more massive halos are too rare to make a significant contribution to the crosscorrelation signal.

\subsection{On the Scale Dependence of the Luminosity-Dependent Cross-Clustering}

For the LRG- $L_{*}$ cross-correlation, Eisenstein et al. (2005a) find a strong dependence on LRG luminosity. The clustering amplitude becomes higher for more luminous LRGs and varies by a factor of up to four over a fac- 

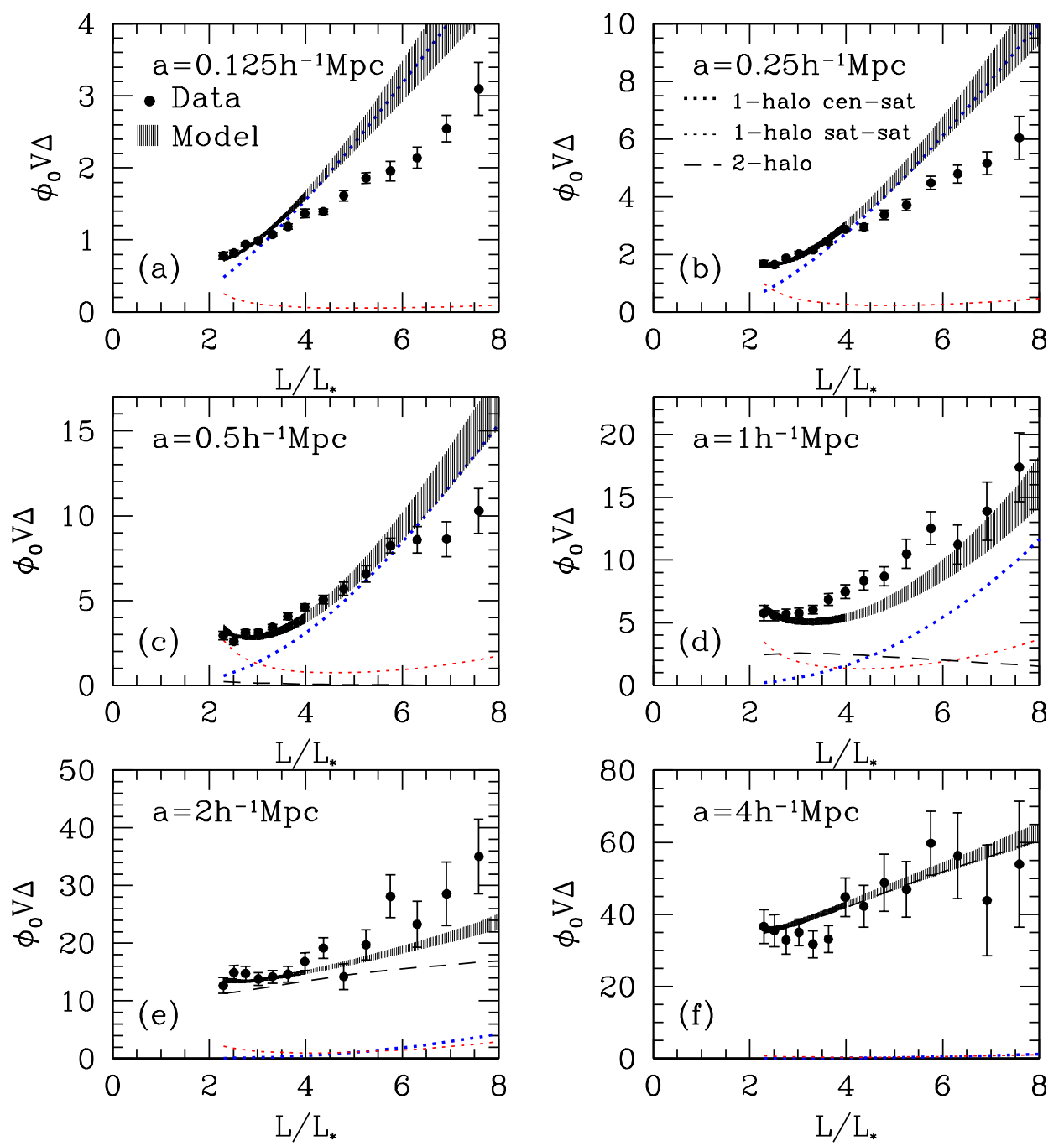

FIG. 5. - The luminosity dependence of the LRG- $L_{*}$ cross-correlation functions at different scales. The six panels correspond to (proper) scales $a=0.125$ to $4 h^{-1} \mathrm{Mpc}$, respectively, as labeled in each panel. The quantity $\phi_{0}=2.267 \times 10^{-2} h^{3} \mathrm{Mpc}^{-3}$ is the proper number density of $L_{*}$ galaxies at the mean redshift $z \sim 0.3$, and $V=3\left(2 \pi a^{2}\right)^{3 / 2}$ is the effective volume for the window function [see eq. [2]]. In each panel, the shaded regions are predictions from the modeling results. Since the model predictions are based on clustering information up to only $\sim 4 L_{*}$, the shaded regions below and above $4 L_{*}$ are model interpolations and extrapolations, respectively. The data points with error bars are the measurements in Eisenstein et al. (2005a). Thick (thin) dotted curves are contributions from central (satellite) LRGs paired with $L_{*}$ galaxies within common halos (calculated from the best-fit HOD model), and dashed curves, which can be clearly seen in panels $(d)-(f)$, represent the two-halo pair contribution.

tor of four in LRG luminosity (see their Fig. 2). Furthermore, the clustering amplitude increases more strongly with luminosity at smaller scales. We now show that these complex trends can be largely explained by the HOD results described above. We note that the HOD results are based on LRGs with luminosity $L<4 L_{*}$, and while the LRG- $L_{*}$ cross-correlation in Eisenstein et al. (2005a) is measured up to an LRG luminosity of $8 L_{*}$, we focus on the $L<4 L_{*}$ results. We emphasize that we do not intend to explain the data points for $L>4 L_{*}$, for which we extrapolate our results. Our purpose here is to give a qualitative interpretation of the scale-dependent luminosity dependence of the cross-correlation between LRGs and $L_{*}$ galaxies.

From our modeling results, the HOD for LRGs in nar- row luminosity bins can be readily constructed, similar to what we do for the $-21.7<M_{g}<-21.2$ sample. We do not refit auto-correlation functions of different LRG samples; rather, we apply the value of $\sigma_{M_{g}}$ and the scaling $L_{c} \propto M^{p}$ derived from the faint and bright luminositythreshold samples and interpolate (or extrapolate) the satellite occupation function from these samples. Figure 5 compares the predicted dependence of the crosscorrelation on LRG luminosity to the observed one. The plotted quantity $\phi_{0} V \Delta$ is the average excess number of $L_{*}$ galaxies around an LRG in an effective spherical volume of $V$ (see eq. 11). We have already shown (Fig. 4) that our HOD model reproduces the overall scale dependence of the cross-correlation accurately. Figure 5 shows that the model also captures the trend of stronger lu- 
minosity dependence at smaller scales. Since the prediction is mostly based on modeling LRGs in the luminosity range of $2-4 L_{*}$, it becomes less accurate at higher luminosities, where it is essentially an extrapolation.

Close inspection of the observed points shows that, at the smallest scales, the clustering amplitude rises steeply and steadily from $\sim 2 L_{*}$ to $\sim 8 L_{*}$; while on larger scales, the luminosity dependence in the $2 L_{*}-4 L_{*}$ range is relatively flat, before steepening at higher luminosities. In addition, the overall luminosity dependence is weaker at larger scales. An intuitive understanding of these features can be built from our HOD modeling results.

At very small scales, over all the LRG luminosity range, the cross-correlation signal is dominated by central LRG paired with satellite $L_{*}$ galaxies. The thick dotted curves in Figure 5 show this component from the model. The slope of this component is estimated as follows. Approximating the mean occupation function of central LRGs in a narrow luminosity bin as a Dirac- $\delta$ function, the twopoint cross-correlation function at a separation $r$ between $L_{*}$ galaxies and LRGs is simply proportional to the pair number $\left\langle N_{*}(M)\right\rangle f(r ; M)$, where $\left\langle N_{*}(M)\right\rangle$ is the mean occupation function of $L_{*}$ galaxies in halos of mass $M$ and $f(r ; M)$ is the fraction of $L_{*}$ galaxies located at a radius $r$ from the central LRG in halos of mass $M$. The function $f$ is just the distribution profile of $L_{*}$ galaxies, $f(r ; M)=\rho(r ; M) r^{2} / \int \rho(r ; M) r^{2} d r$. Using a power law to approximate the inner profile, $\rho(r ; M) \propto\left(r / R_{\text {vir }}\right)^{\gamma}$, we have $f(r ; M) \propto R_{\text {vir }}^{-(3+\gamma)} \propto M^{-(1+\gamma / 3)}$, where $R_{\text {vir }}$ is the virial radius of the halo. Since $\left\langle N_{*}(M)\right\rangle \propto M^{\alpha_{h}}$, we see that the cross-correlation amplitude $\Delta \propto M^{\alpha_{h}-(1+\gamma / 3)}$. Noting that the central luminosity $L \propto M^{p}$, the crosscorrelation amplitude has the dependence on luminosity as $\Delta \propto L^{\left(\alpha_{h}-1-\gamma / 3\right) / p}$. Inserting typical values of the model results, $\alpha_{h}=1.49, \gamma=-1$ (inner NFW profile), and $p=0.66$ to the expression, we obtain $\Delta \propto L^{1.2}$, close to the observational result, which is roughly $\Delta \propto L^{1.1}$.

On larger scales (but still within the regime of one-halo pair domination), the contribution from satellite LRGs paired with satellite $L_{*}$ galaxies starts to show up at the low luminosity end, as can be seen at scales 0.25 and $0.5 h^{-1} \mathrm{Mpc}$ in Figure 5. At a fixed scale, most satellitesatellite pairs come from halos of a narrow mass range. As the luminosity in consideration increases, the occupation number of satellite LRGs at this halo mass decreases, leading to a decreasing contribution to the clustering amplitude from satellite-satellite pairs. The opposing dependences of clustering contributions from central LRGs and satellite LRGs on luminosity flattens the overall luminosity dependence at the low luminosity end, a feature seen in the observed clustering.

On much larger scales (e.g., $4 h^{-1} \mathrm{Mpc}$ ), the two-halo pairs dominate the cross-correlation between LRGs and $L_{*}$ galaxies, and the signal is proportional to the largescale bias factor of LRGs. If the LRG bias factor is approximated by the halo bias factor, the luminositydependent clustering simply reflects the dependence of the halo bias factor on the halo mass. Around $M \sim$ $10^{14} h^{-1} M_{\odot}$, the halo bias factor can be approximated by a power law with index $\alpha_{b} \sim 0.3-0.4$. This, together with the relation between central luminosity and halo mass $L \propto M^{p}(p \sim 0.66)$, gives a luminosity dependence of the cross-clustering following roughly as $L^{0.5}$, which agrees well with the observed trend.

In modeling the LRG- $L_{*}$ cross-correlation, we make the assumption that there is no correlation between the occupation numbers of LRGs and $L_{*}$ galaxies inside the same halo. Loosing this assumption would lead to changes in the contributions from one-halo centralsatellite and satellite-satellite pairs. These changes would be at the level of fine details, and the above picture of the interplay among the three components for interpreting the scale-dependent luminosity dependence of the cross-correlation would remain valid.

\section{SUMMARY AND DISCUSSION}

We have modeled the two-point auto-correlation functions of LRGs and the two-point cross-correlation functions between LRGs and $L_{*}$ galaxies in the SDSS, within the HOD framework, obtaining results on the mean relation between central LRG luminosity and halo mass, the dispersion about this relation, the slope and amplitude of the satellite occupation function, and the abundance of $L_{*}$ galaxies in massive halos.

The continuous rise toward small scales of two-point auto-correlation functions of LRGs implies that not all LRGs are the bright central galaxies in galaxy groups or clusters; a fraction of LRGs must be satellites to produce small scale, one-halo pairs. However, the satellite fraction is small and decreases with the LRG luminosity, e.g., $\sim 5-6 \%$ for $M_{g}<-21.2$ and $\sim 2-3 \%$ for $M_{g}<-21.8$ based on the HOD modeling. The characteristic minimum host halo mass of central LRGs $\left(M_{\min }\right.$, at which $50 \%$ of halos host a galaxy above the luminosity threshold) is a few times $10^{13} h^{-1} M_{\odot}$ and increases with LRG luminosity.

Zehavi et al. 2005b; see also Zheng. Coil. \& Zehavi 2007) found a ratio $M_{1} / M_{\min } \sim 20$ between the halo mass required to host a satellite above a luminosity threshold and the mass required to host a central galaxy above the same threshold. For these LRG samples, which populate higher mass halos and have a median redshift $z \sim 0.3$, we find a smaller ratio, $M_{1} / M_{\min } \sim 10 . \mathrm{A}$ similar drop is seen for the brightest sample $\left(M_{r}<\right.$ -22 ) in Zehavi et al. (2005b), which has a mean redshift $\sim 0.16$. The decrease of the scaling factor reflects the balance between accretion and destruction of satellites (Zentner et al. 2005) - massive halos assemble more recently and their satellites have less time to merge with the central galaxy. The relatively higher redshift of the samples further strengthens this effect.

The HOD of LRGs has been inferred using different methods in several recent investigations. To compare our results with others, one needs to pay attention to the differences in the sample definition, the underlying assumptions, and the modeling details. Mandelbaum et al. (2006) present a mass determination of host halos for two SDSS LRG samples based on galaxy-galaxy lensing measurements. The construction of their LRG samples is not identical to ours, but the halo masses determined from their two samples appear to be consistent with those of the two luminosity-threshold LRG samples we model. The galaxy lensing directly measures the halo masses, while our results come from the mass distribution of halos and the galaxy assignment required to reproduce the observed clustering. The agreement between the two results is therefore encouraging. Wake et al. (2008) model 
the projected two-point correlation functions for LRGs in the 2dF-SDSS LRG and QSO survey with a threeparameter model. For the SDSS $z=0.21$ LRG sample, their inferred mean occupation function is in general agreements with ours. Blake et al. (2008) perform HOD modeling of the two-point angular correlation function of $0.4<z<0.7$ SDSS LRGs with photometric redshifts. Their parameterization is a slight variation of our fiveparameter model presented in Appendix B. For samples with similar number densities, their inferred halo mass scales, cutoff widths of central galaxy occupation function, and high mass slopes of satellite occupation function are close to what we obtain. Kulkarni et al. (2007) constrain the HOD of SDSS LRGs with redshiftspace two-point and three-point correlation functions, by comparing the measurements to those from mock catalogs generated through populating halos identified in $N$-body simulations. They use a three-parameter HOD description and assume no velocity bias. They find that redshift-space three-point correlation functions favor a lower high mass slope $(\sim 1.4)$ for the satellite occupation function. Since the $z=0$ outputs of $\sigma_{8}=0.9$ simulations are used in their modeling while the median redshift of LRGs is about 0.3 , the effective $\sigma_{8}$ in their modeling is about 1.05. From Appendix B (eq. [B3]), we see that the high mass slope from our modeling is 1.5 for such a high $\sigma_{8}$, which is close to the value favored by Kulkarni et al. (2007). However, we note that they use quite a different halo definition than ours (with a much lower overdensity threshold), which complicates the comparison. A more detailed comparison of the LRG modeling results with different methods and samples can be found in Brown, Zheng. White, et al. (2008).

The inferred high mass slopes of the LRG occupation functions tend to be substantially larger than unity, either from our results or others (e.g., Blake et al. 2008; Kulkarni et al. 2007; Wake et al. 2008). This appears to differ from observational inferences and theoretical predictions for low luminosity samples (e.g., Zehavi et al. 2005b; Zheng. Coil. \& Zehavi 2007; Kravtsov et al.|2004; Zheng et al. 2005; Conroy et al. 2006). Is the steep slope of the inferred LRG occupation function a true feature or merely a result of modeling imperfections? First of all, the steep slope should not be a result of any restriction in our HOD parameterizations. Our five-parameter HOD model (Appendix B) introduces a cutoff in $\left\langle N_{\text {sat }}(M)\right\rangle$ at the low mass end so that the connection between the high mass end slope and that at $\left\langle N_{\text {sat }}(M)\right\rangle \sim$ a few is broken. For the fainter LRG sample, the constraint on the slope up to $M \sim 2 \times 10^{15} h^{-1} M_{\odot}$ remains tight even with our more flexible parameterization [see Fig. [1 $(b)$ ]. In the LRG survey volume, there are not many halos that are more massive than $10^{15} h^{-1} M_{\odot}$. The true halo mass function in this volume can therefore deviate from the theoretical one used in the modeling. In Appendix $\mathbb{C}$ we show that this fluctuation in the halo mass function does not seem to introduce any systematic biases in model fitting as it is already reflected in the covariance matrix of the data. To determine which features of the data drive the steep slope, we fit the faint LRG sample with the five-parameter model after excluding some data points and find that the two data points at $r_{p} \sim 1.7 h^{-1} \mathrm{Mpc}$ and $2.7 h^{-1} \mathrm{Mpc}$ play a large role - if they are excluded, the slope drops from 1.8 to 1.6 (for $\sigma_{8}=0.8$ ). These scales are in the one-halo to two-halo transition region where the model is sensitive to the treatments of halo exclusion and scale-dependent halo bias. A more accurate scale-dependent halo bias with halos defined by spherical over-density ( $\mathrm{SO}$ ) could lead to a somewhat lower value of the high mass end slope (J. L. Tinker, private communication). Reid \& Spergel (2009) constrain the LRG HOD with the counts-in-cylinders multiplicity function and the correlation function through populating halos in a simulation. They obtain a good fit by using SO halos and the high-mass end slope is found to be close to unity. Compared to friends-of-friends (FoF; Davis et al. 1985) finder, the SO halo finder does not have the problem of linking two halos by a thin bridge of particles. We plan to pursue analytic models of galaxy clustering based on SO halo properties (Tinker et al. 2008) in future work.

Because of the steep high mass slope, our model fits predict that massive clusters should host multiple LRGs. For example, the LRG occupation number of a $2 \times 10^{15} h^{-1} M_{\odot}$ cluster would be about ten. Such a prediction can be tested with a cluster catalog if the mass can be determined. Using a sample of X-ray selected galaxy clusters at $0.2<z<0.6$, Ho et al. (2007) assess cluster membership for LRGs based on their photometric redshifts and assign halo mass based on X-ray luminosity. They define halos as objects with mean density of 200 times the critical density rather than the mean background density as we do, and they assume $\Omega_{m}=0.238$. After correcting the differences in the halo definition and cosmology, our HOD result for the faint LRG sample matches theirs in the regime of $\langle N(M)\rangle \sim$ a few. (However, near the cutoff mass they find a much larger occupation number, while we have $\langle N(M)\rangle<1$.) In their catalog, there are only two very massive clusters with masses of $\sim 8 \times 10^{14} h^{-1} M_{\odot}$ and $\sim 1.1 \times 10^{15} h^{-1} M_{\odot}$ (corrected to be consistent with our halo definition), and the (corrected) numbers of LRG members are about 8 and 14 , respectively. We also performed a rough calculation to associate LRGs in the faint sample to MaxBCG clusters (Koester et al. 2007) in the overlapped sky region and redshift range. The mass of each cluster is estimated from the total number of MAIN galaxies inside the virial radius, with calibration by weak lensing (Sheldon et al. 2007). For each cluster, we infer the halo radius and velocity dispersion from the halo mass corrected to match our halo definition. LRGs that fall in the projected halo radius and three times the velocity dispersion along the line-of-sight direction are assigned as cluster members (with no completeness and edge correction applied). The number of LRG members for clusters more massive than $6 \times 10^{14} h^{-1} M_{\odot}$ is found to range from 0 to 7 . The predicted number of LRGs in high mass halos (Fig. 11) appears approximately consistent with the estimates from clusters. However, given the uncertainties in the mass estimator and the small number statistics, more work is needed to test our derived values of the high mass slope.

By modeling clustering of LRGs with different luminosities, we infer how the mean luminosity of central galaxies changes with the mass of their host halos, $L_{c} \propto M^{p}$ with $p \sim 0.46-0.51$ for $M \sim 10^{14} h^{-1} M_{\odot}$. This is consistent with the relation $M \propto L^{2}$ found by 

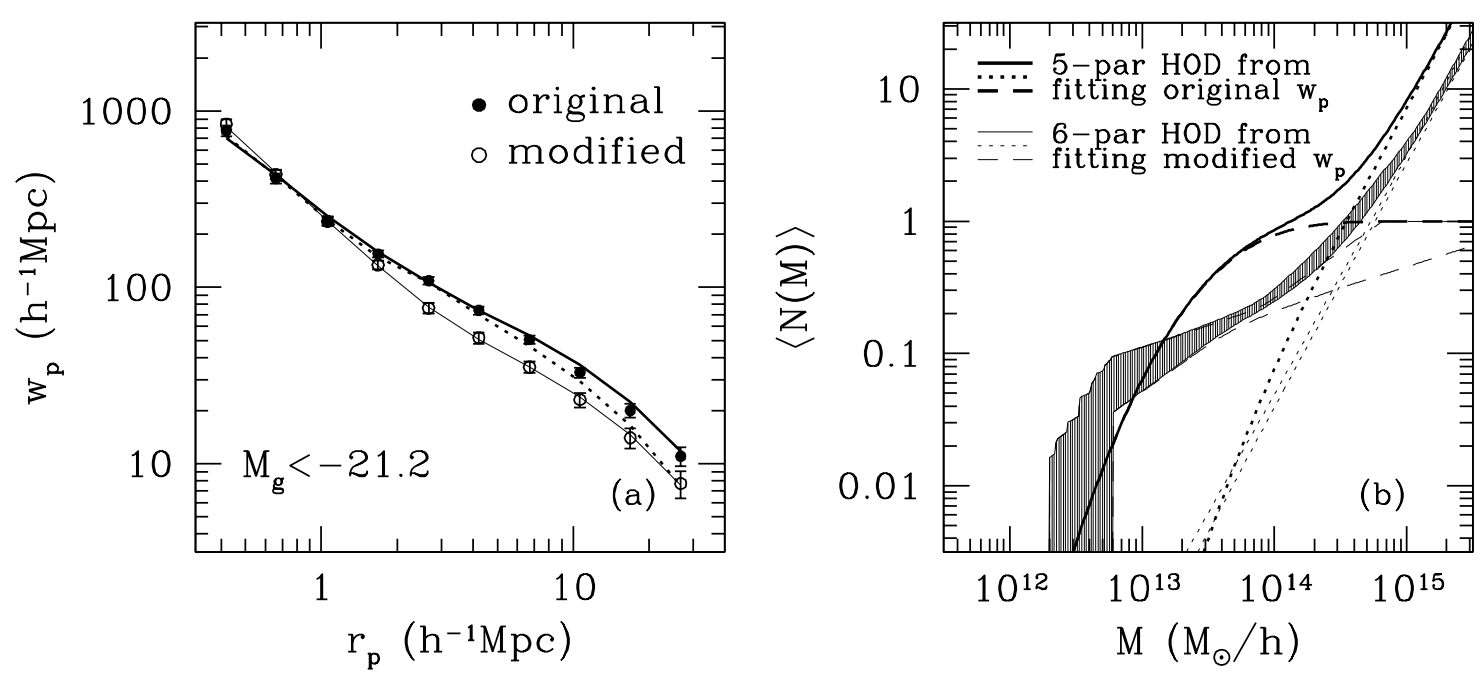

FIG. 6.- Modified projected two-point correlation function and six-parameter HOD fit. Panel $(a)$ shows the original $w_{p}\left(r_{p}\right)$ data points (filled circles) and the modified ones (open circles), which approximate those predicted by the semi-analytic model of Bower et al. (2006) (see Almeida et al. 2008). The thick curve is the best fit to the original data from the five-parameter HOD model and the dotted curve is that from the HOD model presented in $\S 3.1$. The thin curve is the best fit to the modified data from the six-parameter HOD model (see the text). The two curves are best HOD model fits. Panel (b) shows the best fit mean occupation functions. The thick curves are from fitting the original $w_{p}\left(r_{p}\right)$ with the five-parameter model and the thin ones are the $\Delta \chi^{2}<1$ envelopes of mean occupation functions from fitting the modified $w_{p}\left(r_{p}\right)$ with a six-parameter model (see the text) and and those for all galaxies are shaded. Dashed, dotted, and solid curves are for central, satellite, and all galaxies, respectively.

Mandelbaum et al. (2006) based on galaxy-galaxy lensing measurements. Padmanabhan et al. (2009) model the clustering of a sample of photometrically selected SDSS LRGs that are fainter than those in the spectroscopic samples we use. Combining their HOD modeling results with ours for LRGs at $z \sim 0.3$, we find that the value of $p$ is in the range of $0.4-0.5$ over a larger range of LRG luminosity. From a study of the halo occupation statistics of galaxies using galaxy groups identified in the Two Degree Field Galaxy Redshift Survey (2dFGRS; Colless et al. 2001), Yang et al. (2005) infer a $L_{c}-M$ relation that is well described by a broken power law with $p \sim 2 / 3$ and $1 / 4$ below and above $10^{13} h^{-1} M_{\odot}$, respectively. The inferred $L_{c}-M$ relation by Vale \& Ostriker (2004) from matching the luminosity function from the 2dFGRS with the theoretical subhalo mass function has a similar high-mass slope $p \sim 0.28$. More et al. (2009) constrain the high-mass slope to be $0.28_{-0.09}^{+0.07}$ for $z<0.072$ SDSS galaxies based on satellite kinematics. The highmass slope extrapolated from the HOD modeling results of SDSS MAIN galaxies (Zheng, Coil, \& Zehavi 2007) is also about 0.3 . The value we obtain at the high-mass end from modeling the LRG clustering differs significantly from these results. It is interesting to see whether such a difference can be explained by the difference in the galaxy samples. The 2dFGRS luminosity is in the $b_{J}$ band, while we use the SDSS $g$-band luminosity. Since the wavelength coverages of these two bands are close, it seems unlikely that the band difference can cause the apparent discrepancy. The other thing to notice is that the 2dFGRS galaxies and the SDSS MAIN galaxies have a mean redshift $\sim 0.1$ and the LRG galaxies in our analysis are located around redshift 0.3. Could the discrepancy indicate an evolution effect over the intervening $\sim 2$ billion years? Through fitting restframe $B$-band galaxy luminosity functions at different redshifts using a CLF approach, Coorav (2005) finds that the data are compatible with a halo-mass-dependent central galaxy luminosity evolution, with the high mass slope $p$ increasing with redshift. The fitting results of Cooray (2005) imply that $p$ could be as high as 0.5 at $z \sim 0.3$, close to our inferred value.

If we take the inferred values of $p$ at $z \sim 0.3$ from our analysis and at $z \sim 0.1$ from the 2dFGRS studies at face value, they indicate that the luminosity evolution of central bright galaxies depends on halo mass in the sense that either galaxies in more massive halos fade more or the fraction of stars that were assembled into central galaxies from mergers between $z \sim 0.3$ and $z \sim 0.1$ is smaller in more massive halos. Bernardi et al. (2006)'s study of the properties of early-type galaxies in the SDSS as a function of local environment and redshift suggests that star formation in early-type galaxies happens earlier in dense regions and lasts over a shorter time-scale, which implies that central galaxies in more massive halos on average experience star formation at an earlier epoch. Since younger stellar populations fade faster than older ones, this seems to rule out the possibility that luminosity of central galaxies in more massive halos fades more between $z \sim 0.3$ and $z \sim 0.1$. We are left with the possibility that mergers in more massive halos are less efficient in adding stars to the central galaxies, which appears to be consistent with our finding of the drop of $M_{1} / M_{\min }$ and its interpretation based on the competition between accretion and destruction as a function of halo mass. This is also in line with the results of LRG evolution in Brown. Zheng. White, et al. (2008) from HOD modeling of their clustering from $z \sim 0.2$ to $z \sim 1.0$ in the NOAO Deep Wide-Field Survey.

Since LRGs naturally separate out massive halos, our HOD modeling of the two-point cross-correlation functions between LRGs and $L_{*}$ galaxies circumvents the 
usual challenge in constraining the HOD of $L_{*}$ galaxies in massive halos from their two-point auto-correlation function. We show that the cross-correlation data are consistent with the case where the distribution of $L_{*}$ galaxies inside massive halos roughly follows that of the matter at distances greater than $\sim 0.2 h^{-1} \mathrm{Mpc}$ and that the mean number of $L_{*}$ galaxies scales with halo mass as $M^{1.5}$. The slope of the mean occupation function is steeper than what is found for $z \sim 0$ galaxies, which is $\sim 1.10$. There may not be inconsistency between the results. The constraint for the slope for $z \sim 0$ galaxies from auto-correlation function is not sensitive to the occupation in very massive halos, while here from crosscorrelation with LRGs, we have a better constraint on the $L_{*}$ occupation function in massive halos. On the other hand, the selection of $L_{*}$ galaxies and its redshift dependence make it hard to do a precise comparison between results at $z \sim 0$ and at $z \sim 0.3$.

The luminosity dependence of the LRG- $L_{*}$ galaxy cross correlation depends on scale in a rather complex way (Eisenstein et al. 2005a). By separating contributions from pairs of $L_{*}$ galaxies with central and satellite LRGs in common halos and in different halos, our HOD modeling results explain these trends, in a relatively transparent way. At a fixed scale, the luminosity dependence in the cross correlation reflects the luminositydependent HOD of LRGs. As the scale in consideration changes, the relative contributions of the one-halo central-satellite, the one-halo satellite-satellite, and the two-halo LRG- $L_{*}$ pairs to the cross-correlation function vary, which leads to the scale-dependent luminosity dependence of the cross correlation.

Our LRG modeling results establish the relation between massive galaxies and dark matter halos at $z \sim 0.3$, which itself provides useful tests to models of formation of massive galaxies. Almeida et al. (2008) present predictions for properties of LRGs in semi-analytic galaxy formation models. They show that the Bower et al. (2006) model, which is based on the Millennium simulation (Springel et al. 2005), predicts a $z=0.24$ LRG luminosity function that is in good agreement with the observation, although it fails at $z=0.5$. Compared to the measurement, the Bower et al. (2006) model seems to predict the $z=0.24$ LRG two-point correlation function remarkably well on both small and large scales (see their Fig.13). However, the LRG HOD predicted in this model differs significantly from our modeling results presented in this paper: (a) in the Bower et al. (2006) model, LRGs in the fainter sample can reside in halos of $10^{12} h^{-1} M_{\odot}$ (with mean occupation number of $\sim 0.01$ ), much lower than the mass scale we infer; (b) the mass of halos that can on average host one LRG is about $3 \times 10^{14} h^{-1} M_{\odot}$, higher than what we find (see Fig.11 in Almeida et al. 2008 and Fig.16 in Wake et al. 2008); (c) the probability distribution of LRG host halo masses is broad (see Fig.16 in Wake et al. 2008), ranging from $10^{12} h^{-1} M_{\odot}$ to $10^{15} h^{-1} M_{\odot}$, rather than narrowly peaked around a few times $10^{13} h^{-1} M_{\odot}$ as we find (Fig. 1 $d$ ).

Does the discrepancy between the theoretically predicted HOD and our observationally inferred HOD imply that our HOD parameterization is not generic enough to model LRG clustering? To investigate the problem, we modify the five-parameter HOD model (Appendix B) to mimic the shape of the mean occupation function predicted in Bower et al. (2006) model. The parameterization for the satellite mean occupation function remains unchanged (with three parameters). For the central galaxy occupation function, we model it as a linear (in logarithmic space) ramp going from $\left\langle N_{\min }\right\rangle$ at $M_{\text {min }}$ to unity at $M_{u}$ and staying at unity for $M>M_{u}$. In total, this parameterization has six free parameters. With the measured $w_{p}\left(r_{p}\right)$, we find that the best-fit HOD from this six-parameter model (not shown in Figure 6b) closely follows the result from the five-parameter model (shown as thick curves in Figure 6b). Therefore, change in the HOD parameterization does not solve the discrepancy and the five-parameter model is not inadequate in modeling LRG clustering. A close look at Figure 13 in Almeida et al. (2008) shows that the predicted twopoint correlation function does not match the data perfectly - it is $\sim 15 \%$ higher on scales of $0.1-2 h^{-1} \mathrm{Mpc}$ and $\sim 30 \%$ lower on scales larger than $2 h^{-1} \mathrm{Mpc}$ (note that the vertical range of the plot is over eight orders of magnitude). We therefore modify the amplitude of the observed $w_{p}\left(r_{p}\right)$ data points to mimic the predicted correlation function and perform an HOD fit with the six-parameter model (by adopting the predicted number density, which is $10 \%$ lower than the observed one). The results on the mean occupation functions are shown in panel $(b)$ of Figure 6 as thin curves. Note that the shaded region represents the $\Delta \chi^{2}<1$ envelopes and the linear ramp for the central galaxy mean occupation number (thin dashed curves) has not reached unity at the highest mass in the plot. The mean occupation function can extend to halos of mass as low as $2 \times 10^{12} h^{-1} M_{\odot}$ and reaches unity around $4 \times 10^{14} h^{-1} M_{\odot}$, which appears to be approximately consistent with the prediction of the Bower et al. (2006) model. From the above investigations, we conclude that the discrepancy between the theoretically predicted LRG HOD and the observationally inferred LRG HOD reflects the imperfection of the semianalytic galaxy formation model - the 15-30\% discrepancies with observed clustering are real and physically significant - rather than limitations in our HOD parameterization. The results suggest that the mechanism of turning blue galaxies to red in the semi-analytic model is too efficient in halos of a few times $10^{12} h^{-1} M_{\odot}$ and not efficient enough in more massive halos. Our HOD modeling results thus provide important tests to galaxy formation theory.

In combination with passive evolution of LRGs and a halo merger history (e.g., White et al. 2007; Seo et al. 2008), our modeling results can be used to predict the HOD of LRGs and the clustering of LRGs at lower or slightly higher redshifts. Supplemented with corresponding observations at these redshifts, we would be able to test our understanding of the formation and evolution of massive galaxies. Constraints on the HOD of LRGs are also useful for cosmological parameter investigations based on LRG clustering. For example, the most precise measurements of the large scale galaxy power spectrum have come from the SDSS LRG sample (Tegmark et al. 2006; Percival et al.2007), and the principal limitation in interpreting these measurements is the uncertain level of scale-dependent bias between galaxy and matter power spectra in the mildly non-linear regime. With HOD 
constraints like those derived here, this scale-dependent bias can be calculated and corrected (Yoo et al. 2009). As another example, HOD constraints could be combined with galaxy-galaxy lensing measurements of the LRG-mass cross-correlation (R. Mandelbaum et al., in preparation) to improve determinations of $\sigma_{8}$ and $\Omega_{m}$ (Yoo et al. 2006). The role of LRGs in cosmological studies seems destined to grow with surveys that target large numbers of LRGs to measure baryon acoustic oscillations, including AAOmega LRG survey (Ross et al. 2008) and the Baryon Oscillation Spectroscopic Survey (BOSS), part of a proposed successor to SDSS-II. Understanding the evolving relation between LRGs and dark matter halos will be crucial to exploiting their power as cosmological probes and to revealing the physics that governs the formation of the most massive galaxies in the universe.

We are grateful to Jeremy Tinker and Jaiyul Yoo for valuable discussions. We thank Erin Sheldon for providing us with his cluster lensing results and acknowledge the Aspen Center for Physics, where discussions in a stimulating atmosphere led to a more complete presentation of this paper. We thank Tobias Baldauf for pointing out typos in an early draft. Finally, we thank the referee for a careful reading of the paper and for helpful comments that improved the paper.

At an early stage of this work, ZZ was supported by NASA through Hubble Fellowship grants HF-01181.01A, awarded by the Space Telescope Science Institute, which is operated by the Association of Universities for Research in Astronomy, Inc., for NASA, under contract NAS 5-26555. ZZ also acknowledges support from the Institute for Advanced Study through a John Bahcall Fel- lowship. IZ and ZZ acknowledge support from NSF grant AST-0907947 and DW acknowledges support from NSF grant AST-0707985. IZ was further supported by NASA through a contract issued by the Jet Propulsion Laboratory. YPJ is supported by NSFC (10533030), by the Knowledge Innovation Program of CAS (No. KJCX2YW-T05), and by 973 Program (No.2007CB815402).

Funding for the SDSS and SDSS-II has been provided by the Alfred P. Sloan Foundation, the Participating Institutions, the National Science Foundation, the U.S. Department of Energy, the National Aeronautics and Space Administration, the Japanese Monbukagakusho, the Max Planck Society, and the Higher Education Funding Council for England. The SDSS Web Site is http://www.sdss.org/.

The SDSS is managed by the Astrophysical Research Consortium for the Participating Institutions. The Participating Institutions are the American Museum of Natural History, Astrophysical Institute Potsdam, University of Basel, Cambridge University, Case Western Reserve University, University of Chicago, Drexel University, Fermilab, the Institute for Advanced Study, the Japan Participation Group, Johns Hopkins University, the Joint Institute for Nuclear Astrophysics, the Kavli Institute for Particle Astrophysics and Cosmology, the Korean Scientist Group, the Chinese Academy of Sciences (LAMOST), Los Alamos National Laboratory, the Max-Planck-Institute for Astronomy (MPIA), the MaxPlanck-Institute for Astrophysics (MPA), New Mexico State University, Ohio State University, University of Pittsburgh, University of Portsmouth, Princeton University, the United States Naval Observatory, and the University of Washington.

\section{APPENDIX}

\section{ROLE OF HALO MASS SCALES ON THE DEPARTURES FROM A POWER LAW IN THE GALAXY TWO-POINT} CORRELATION FUNCTION

Departures of the galaxy two-point correlation function from a pure power law have been observed for both low and high redshift galaxies (Hawkins et al. 2003; Zehavi et al. 2004; Ouchi et al. 2005; Coil et al. 2006; Lee et al. 2006). In the two luminosity-threshold LRG samples studied in this paper, the departures are also clearly seen, shown as an upturn in the two-point correlation function at small scales (see also Zehavi et al. 2005a). Such departures have been nicely explained by the HOD model as the transition from a regime dominated by one-halo pairs on small scales to that dominated by two-halo pairs on large scales (Zehavi et al. 2004). The strength of the departures of the galaxy two-point correlation function from a pure power law is closely related to the amplitude of the one-halo term, which itself depends on the scatter in the occupation number and the halo mass function (Benson et al. 2000; Berlind \& Weinberg 2002). It would be helpful to gain a better understanding of the key factor that determines the strength of the departures.

For luminosity-threshold samples, there are two mass scales, the characteristic minimum mass $M_{\text {min }}$ of halos that host central galaxies above the luminosity threshold and the mass scale $M_{1}$ of halos that on average host one satellite galaxy above the luminosity threshold. An additional mass scale is the nonlinear mass $M_{\mathrm{nl}}$, marking a transition in the halo abundance from a power-law form to an exponential cutoff at high mass. We can thus identify two ratios that can shape the one-halo term: $M_{1} / M_{\min }$ and $M_{\min } / M_{\mathrm{nl}}$. The $M_{1} / M_{\min }$ ratio affects the shape of the galaxy occupation functions and tells us how quickly the transition from sub-Poisson to Poisson scatter occurs when going to higher halo masses. A smaller $M_{1} / M_{\text {min }}$ increases the importance of one-halo pairs by increasing the satellite fraction and thus results in a higher amplitude of the one-halo term. The $M_{\min } / M_{\mathrm{nl}}$ ratio determines which part of the halo mass function the galaxy sample probes. The slope of the one-halo term reflects the drop of the halo mass function toward high masses. For a galaxy sample that probes the exponential tail of the halo mass function $\left(M_{\mathrm{min}} / M_{\mathrm{nl}} \gtrsim 1\right)$, we expect a steep drop in the one-halo term and thus a sharp upturn around the one-halo to two-halo transition scale.

To figure out the relative importance of $M_{1} / M_{\min }$ and $M_{\min } / M_{\mathrm{nl}}$ on the departures from a power law in the galaxy two point correlation function, we calculate the predicted correlation functions on a $3 \times 3$ grid of $M_{1} / M_{\text {min }}$ and $M_{\min } / M_{\mathrm{nl}}$, as shown in Figure A. In each panel, the short dashed curve is the one-halo term from central-satellite pairs, the long dashed curve is the one-halo term from satellite-satellite pairs, and the dot-dashed curve is the two-halo term. The dotted line is a power-law fit to $w_{p}\left(r_{p}\right)$ in the range of $1-10 h^{-1} \mathrm{Mpc}$ for comparison. The satellite-satellite 


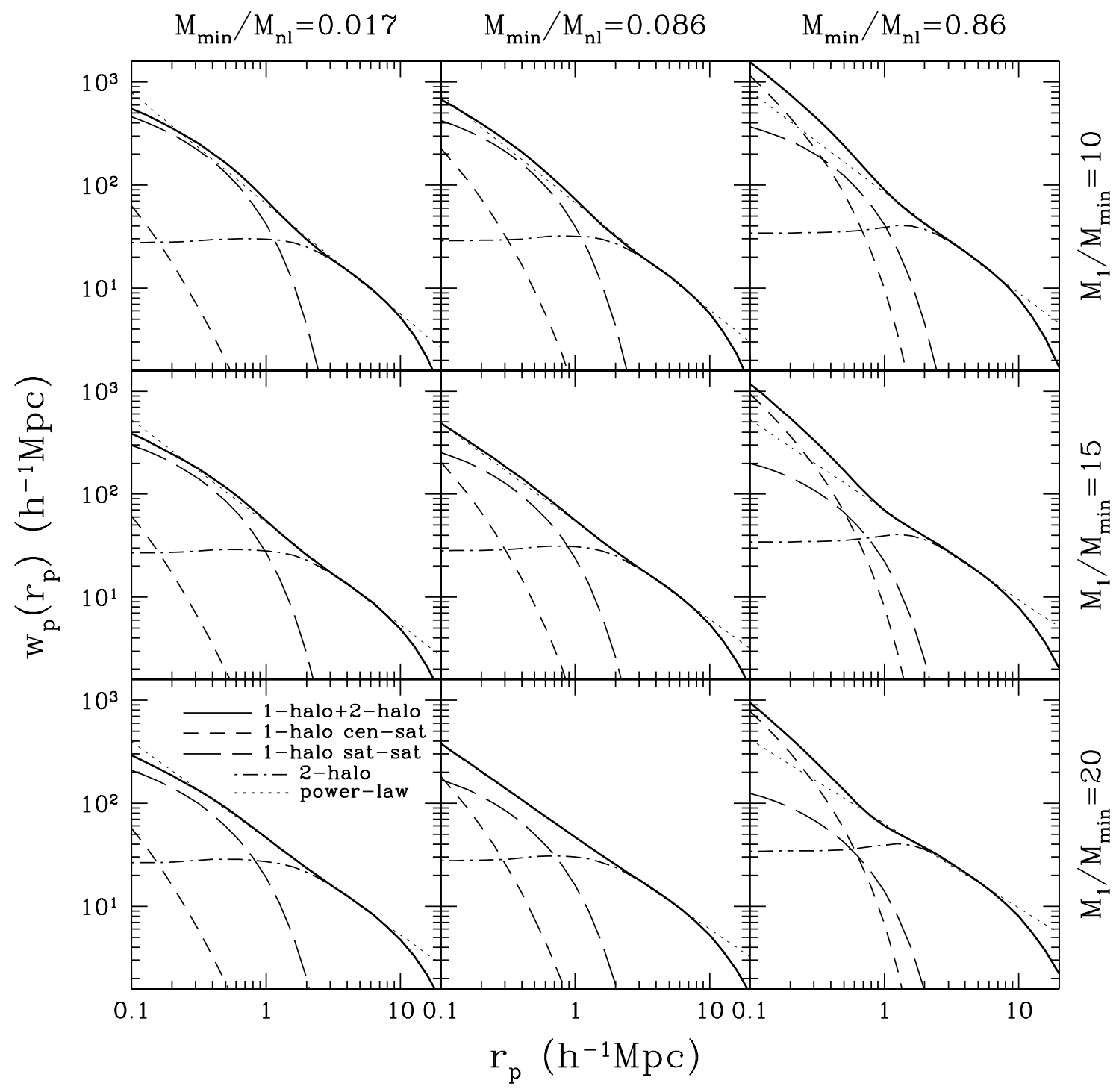

FIG. 7.- Impact of the $M_{1} / M_{\min }$ and $M_{\mathrm{min}} / M_{\mathrm{nl}}$ ratios on departures from a power law in the galaxy two-point correlation function. Each row (column) has the same $M_{1} / M_{\min }\left(M_{\min } / M_{\mathrm{nl}}\right)$ with the value marked on the right (top) of the plot. In each panel, the solid line shows the predicted projected correlation function, $w_{p}\left(r_{p}\right)$. The short and long dashed curves are the one-halo terms from central-satellite and satellite-satellite galaxy pairs, respectively, and the dot-dashed curve is the two-halo term. The dotted line is a power-law fit to $w_{p}\left(r_{p}\right)$ in the range of $1-10 h^{-1} \mathrm{Mpc}$ to guide the eye.

pair contributions are similar at a fixed $M_{1} / M_{\min }$, since $\left\langle N_{\text {sat }}(M)\right\rangle$ is assumed to be proportional to halo mass. In a massive halo of fixed mass, however, the contribution of central-satellite pairs relative to satellite-satellite pairs is larger for a higher $M_{\min } / M_{\mathrm{nl}}$ sample because of its low satellite occupation number. This leads to a larger slope across the central-satellite and satellite-satellite contributions for a higher $M_{\min } / M_{\mathrm{nl}}$ sample, thus a steeper upturn in the correlation function ${ }^{8}$. In more detail, the slope across the central-satellite and satellite-satellite contributions should depend on the $M_{1}$ mass scale, but $M_{1} / M_{\min }$ should not vary by an extremely large factor at least for a threshold galaxy sample. So a dependence on $M_{1}$ can be translated to that on $M_{\min } / M_{\mathrm{nl}}$. When probing the exponential tail of the halo mass function for observed galaxy samples, we expect a steeper slope in both the central-satellite and satellite-satellite one-halo terms. This in turn leads to a more prominent rise at small scales.

From the results, we conclude that overall the $M_{\min } / M_{\mathrm{nl}}$ ratio plays a much more important role than $M_{1} / M_{\min }$ in driving departures from a power law in galaxy two-point correlation functions. That is, a steeper drop in the halo mass function is translated to a steeper radial cutoff in the one-halo term, leading to a stronger inflection where the one-halo and two-halo term join. This explains why the departure is stronger for more luminous galaxy samples (e.g., Zehavi et al. 2005a, b) that probe the high mass end of the halo mass function. This also explains why the departures

${ }^{8}$ We thank Alison Coil, Jeremy Tinker, and Risa Wechsler for helpful discussions that led us to separately investigate the central-satellite and satellite-satellite pair contributions. 

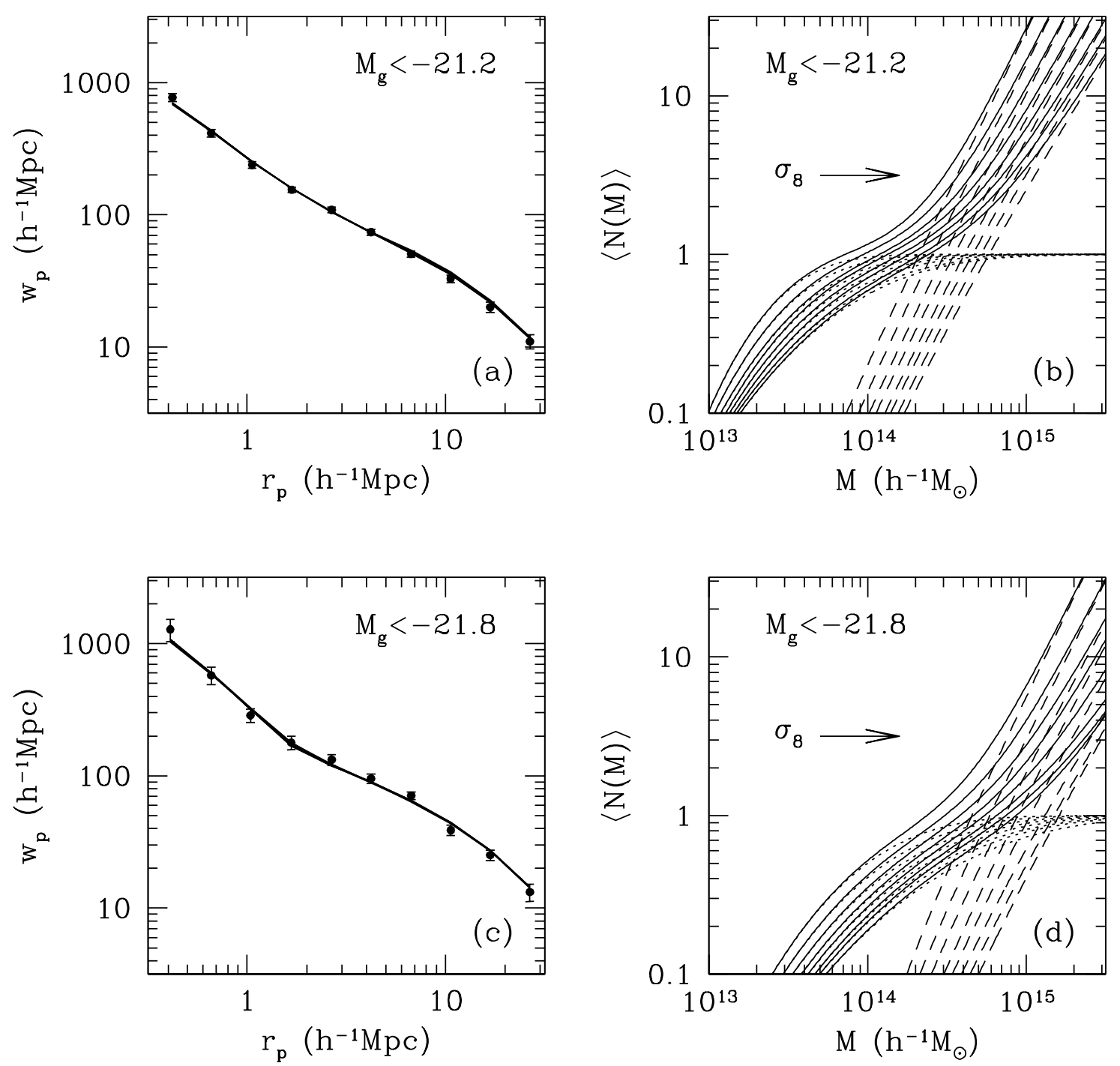

FIG. 8. - Dependence of the LRG HOD on cosmology. Panel $(a)$ shows the measured two-point correlation function (data points with error bars) of the $M_{g}<-21.2$ sample and the best HOD fits (solid curves) for cosmological models differing only in $\sigma_{8}$. Note that the best fits overlap with each other. Panel (b) shows the mean occupation functions (solid curves) corresponding to the best fits, separated into central (dotted) and satellite (dashed) contributions. From left to right, the HODs correspond to $\sigma_{8}$ values increasing from 0.65 to 1.00 with a step-size 0.05 . The two bottom panels are similar, but for the $M_{g}<-21.8$ LRG sample.

are more prominent at higher redshifts (e.g., Ouchi et al. 2005) — the fast drop of the nonlinear mass $M_{\mathrm{nl}}$ toward high redshift makes the observed (bright) galaxies more likely to probe the exponential tail of the halo mass function. A more detailed theoretical investigation of the departure from a power law in the two-point correlation function of galaxies and its dependence on galaxy properties will be presented elsewhere.

\section{LRG HODS WITH A FIVE-PARAMETER MODEL AND DEPENDENCE ON COSMOLOGY}

The LRG clustering modeling in the main text assumes a flexible HOD parameterization, which reveals details of the constraining power of the two-point correlation functions on the HOD. In general, the HODs for luminosity-threshold samples predicted by galaxy formation models can be well described by a less flexible (i.e., more restricted) parametric form. The mean occupation function is well characterized by a step-like function for central galaxies and a power lawlike function for satellite galaxies (Kravtsov et al. 2004; Zheng et al. 2005). We present here the modeling results for the two luminosity-threshold LRG samples with an alternative, five-parameter HOD model and give their dependence on cosmology. This set of results would be useful for comparisons with other work and for making mock catalogs in a wide range of cosmological models.

The mean occupation function of a luminosity-threshold LRG sample, being the sum of central and satellite mean 
occupation functions, is parameterized as (see Zheng et al. 2005; Zheng, Coil, \& Zehavi 2007)

$$
\langle N(M)\rangle=\frac{1}{2}\left[1+\operatorname{erf}\left(\frac{\log M-\log M_{\min }}{\sigma_{\log M}}\right)\right]\left[1+\left(\frac{M-M_{0}}{M_{1}^{\prime}}\right)^{\alpha}\right],
$$

where erf is the error function

$$
\operatorname{erf}(x)=\frac{2}{\sqrt{\pi}} \int_{0}^{\mathrm{x}} e^{-t^{2}} d t
$$

The distribution of the occupation number of central galaxies and satellite galaxies are assumed to follow the nearestinteger and Poisson distributions, respectively, as usual. The five free parameters are the mass scale $M_{\text {min }}$ and width $\sigma_{\log M}$ of the cutoff profile for the mean occupation function of central galaxies and the cutoff mass scale $M_{0}$, normalization $M_{1}^{\prime}$, and high mass slope $\alpha$ of the mean occupation function of satellite galaxies.

We vary the normalization $\sigma_{8}$ (at $z=0$ ) of the matter fluctuation power spectrum from 0.65 to 1.00 with a step-size 0.05. Other cosmological parameters are assumed to be $\left(\Omega_{m}, \Omega_{\Lambda}, \Omega_{b}, n_{s}, h\right)=(0.24,0.76,0.04,0.95,0.73)$. We perform an MCMC run for each LRG sample under each cosmological model and obtain the marginalized distribution for each of the five HOD parameters.

In general, for a higher $\sigma_{8}$, the nonlinear mass increases and there are more high mass halos. To conserve the galaxy number density, the cutoff mass scale $M_{\min }$ needs to increase. The increase in $M_{\min }$ turns out to be slower than that in $M_{\mathrm{nl}}$, which leads to a lower halo bias that is necessary to maintain the large scale clustering of galaxies (i.e., the square of $\left.b_{g} \sigma_{8}\right)$. The width $\sigma_{\log M}$ of the cutoff increases so that some LRGs are populated to lower mass halos to further adjust the large scale bias factor and the galaxy number density. The mean occupation function of satellites also responds to the $\sigma_{8}$ change to match the small scale clustering amplitude: the mass scale $M_{1}^{\prime}$ increases and the slope $\alpha$ decreases with increasing $\sigma_{8}$. For a large range of $\sigma_{8}$, our HOD modeling yields almost identical best fits to the data points, similar to what is found and discussed in Zheng \& Weinberg (2007).

While the dependence of $\log M_{\min }, \sigma_{\log M}, \log M_{0}, \log M_{1}^{\prime}$ or $\alpha$ on $\sigma_{8}$ appears to be quite close to linear, we fit the results by adding a quadratic term for a better accuracy. The HOD parameters for the $M_{g}<-21.2$ LRG sample are

$$
\begin{aligned}
\log M_{\min } & =13.673+1.419\left(\sigma_{8}-0.8\right)-1.706\left(\sigma_{8}-0.8\right)^{2}, \\
\sigma_{\log M} & =0.621+0.908\left(\sigma_{8}-0.8\right)-0.935\left(\sigma_{8}-0.8\right)^{2}, \\
\log M_{0} & =12.339+0.658\left(\sigma_{8}-0.8\right)+9.206\left(\sigma_{8}-0.8\right)^{2}, \\
\log M_{1}^{\prime} & =14.533+1.248\left(\sigma_{8}-0.8\right)-1.394\left(\sigma_{8}-0.8\right)^{2}, \\
\alpha & =1.832-1.326\left(\sigma_{8}-0.8\right)+0.523\left(\sigma_{8}-0.8\right)^{2} .
\end{aligned}
$$

The typical $1-\sigma$ uncertainties for the five HOD parameters are 0.06, 0.07, 0.6, 0.025, and 0.08, respectively. The HOD parameters for the $M_{g}<-21.8 \mathrm{LRG}$ sample are

$$
\begin{aligned}
& \log M_{\min }=14.304+1.694\left(\sigma_{8}-0.8\right)-1.810\left(\sigma_{8}-0.8\right)^{2} \text {, } \\
& \sigma_{\log M}=0.797+0.761\left(\sigma_{8}-0.8\right)-0.614\left(\sigma_{8}-0.8\right)^{2}, \\
& \log M_{0}=12.491-1.476\left(\sigma_{8}-0.8\right)+29.983\left(\sigma_{8}-0.8\right)^{2} \text {, } \\
& \log M_{1}^{\prime}=14.946+1.616\left(\sigma_{8}-0.8\right)-0.712\left(\sigma_{8}-0.8\right)^{2} \text {, } \\
& \alpha=1.717-0.589\left(\sigma_{8}-0.8\right)-7.437\left(\sigma_{8}-0.8\right)^{2} .
\end{aligned}
$$

The typical $1-\sigma$ uncertainties for the these five HOD parameters are 0.06, 0.055, 0.7, 0.1, and 0.4, respectively. All the masses are in units of $h^{-1} M_{\odot}$.

For results with cosmological models with $\Omega_{m}$ different than 0.24 , one only needs to change the three mass scales by a factor of $\Omega_{m} / 0.24$ (see Zheng et al. 2002; Zheng \& Weinberg 2007). This scaling assumes that the shape of the linear power spectrum stays fixed, with a change in $h$ or $n_{s}$ compensating the impact of changing $\Omega_{m}$. Therefore, the solutions here cover a wide range of cosmological models in the $\left(\Omega_{m}, \sigma_{8}\right)$ plane.

\section{HALO NUMBER FLUCTUATION IN THE LRG SURVEY VOLUME AND THE COVARIANCE MATRIX}

In our HOD modeling of the LRG two-point correlation functions, we use a theoretical halo mass function (Jenkins et al. 2001), which is a fitting formula based on $N$-body simulations. In the LRG survey volume, which is $0.72 h^{3} \mathrm{Gpc}^{-3}$ (Eisenstein et al. 2005b) , fluctuations in the number of massive halos (e.g., with mass above $\left.10^{15} h^{-1} M_{\odot}\right)$ are expected. Since LRGs reside in massive halos, the details of their small scale clustering may be sensitive to such fluctuations. In this Appendix, we investigate whether these fluctuations introduce any systematic effect on the HOD modeling.

Jing et al. (2007) performed $N$-body simulations with $1024^{3}$ particles in a box of $1800 h^{-1} \mathrm{Mpc}$ on a side. For our investigation, we make use of the catalog of massive halos $\left(>10^{15} h^{-1} M_{\odot}\right)$ identified in the $z=0.274$ outputs from four independent simulations. The volume of each realization is divided into eight octants. Each octant has a volume similar to the LRG survey volume, so altogether we have 32 sub-volumes to investigate the mass function variations. 

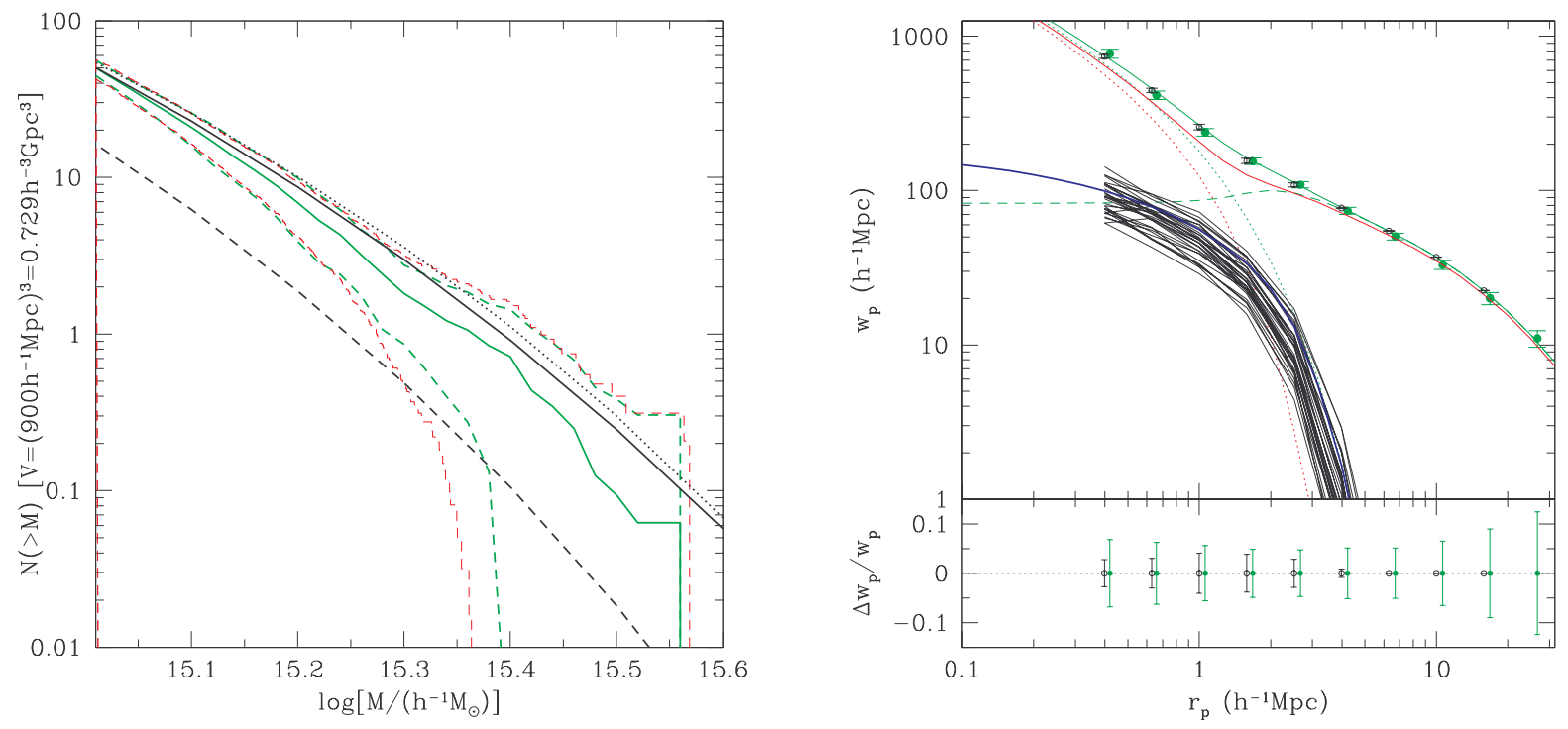

Fig. 9. - (Left): Halo mass function and its scatter from the Jing et al. (2007) simulation, for a volume similar in size to the LRG survey volume. The green solid curve is the mean halo mass function in the simulation, the green dashed curves mark the scatter and the red dashed curves denote a Poisson scatter around the mean. The black solid, dotted, and dashed curves are the Jenkins, Sheth-Tormen, and Press-Schechter mass functions, respectively (see the text). (Right): Contribution of LRGs in massive halos to small-scale $w_{p}$ and the variation due to the fluctuation of halo mass function in a finite volume. The green solid curve is the best fit from a five-parameter HOD model to the measurements (green points). The green dotted and dashed curves are the one-halo and two-halo terms, respectively. The red solid curve is $w_{p}$ calculated by excluding halos more massive than $10^{15} h^{-1} M_{\odot}$. The dotted red curve shows the one-halo term from halos below $10^{15} h^{-1} M_{\odot}$, and the blue curve shows it for halos above this value. The black solid curves are the one-halo term contributions from halos more massive than $10^{15} h^{-1} M_{\odot}$ in each of the 32 simulation subvolumes that have the same size as the LRG survey volume. The bottom panel shows the comparison of the diagonal jackknife errors and uncertainties introduced by the fluctuation of the number of massive halos in the LRG survey volume. See text for more details.

The left panel of Figure $\mathrm{C}$ shows the fluctuation of the number of massive halos among the 32 sub-volumes. The green solid curve is the mean halo mass function in a sub-volume and the green dashed curves mark the scatter around the mean. The scatter turns out to closely follow that of a Poisson distribution (the two dashed red curves). The fluctuation in the number of halos is about $15 \%$ for $M>10^{15} h^{-1} M_{\odot}$ and increases with halo mass. For comparison, we also plot three frequently used theoretical functions for the same cosmological model assumed in the simulation. The Jenkins mass function (Jenkins et al. 2001), shown as the black solid curve, gives a good description of the mean halo mass function at $10^{15} h^{-1} M_{\odot}$ and becomes a factor of two higher at $2 \times 10^{15} h^{-1} M_{\odot}$. The Sheth-Tormen mass function (Sheth \& Tormen 1999; black dotted curve) is slightly higher than the Jenkins mass function, while the PressSchechter mass function (Press \& Schechter 1974; black dashed curve) underestimates the mass function by a factor of five in the mass range considered here.

The right panel of Figure $\mathrm{C}$ shows the effect of the fluctuation in the number of massive halos on the small scale clustering of the $M_{g}<-21.2$ LRGs. The green solid curve is the best fit to the measured $w_{p}$ (green points) from the five-parameter HOD model (Appendix B) with the same cosmological model as used in the simulation. The green dotted and dashed curves are the contributions from the one-halo and two-halo term, respectively. With the best-fit HOD model fixed, the red solid curve show the predicted $w_{p}$ when only keeping halos less massive than $10^{15} h^{-1} M_{\odot}$ in the calculation. The red dotted curve is the one-halo term from halos less massive than $10^{15} h^{-1} M_{\odot}$.

The blue solid curve is the mean contribution to the one-halo term from halos of $M>10^{15} h^{-1} M_{\odot}$. The fluctuation in the number of massive halos would lead to a fluctuation around this mean contribution. To see this, we populate the massive halos in the 32 sub-volumes according to the best-fit HOD model and measure the one-halo $w_{p}$ in each subvolume. Black solid curves show the measurements for individual sub-volumes. Such a fluctuation in the one-halo term introduces uncertainties in the small scale clustering. By adding the one-halo term from halos with $M<10^{15} h^{-1} M_{\odot}$ (red dotted), that from halos above $10^{15} h^{-1} M_{\odot}$ in each sub-volume (black solid) and the two halo-term (green dashed), we obtain the black points with error bars reflecting the fluctuation in the number of massive halos. On small scales, these error bars appear to have similar (somewhat smaller) amplitude to those from the diagonal elements of the covariance matrix (see the comparison in the lower panel), which is computed through jackknife technique.

We see that the fluctuation of the number of massive halos in the LRG survey volume can be large, e.g., $\sim 15 \%$ for $M>10^{15} h^{-1} M_{\odot}$ halos and $\sim 50 \%$ for $M>2 \times 10^{15} h^{-1} M_{\odot}$ halos. This introduces a fluctuation in the small scale clustering of LRGs. However, our investigation suggests that such a fluctuation in $w_{p}$ should be correctly reflected in the jackknife covariance matrix. Therefore, it is sufficient to use the mean mass function in modeling the two-point correlation functions of LRGs.

\section{REFERENCES}

Almeida, C., Baugh, C. M., Wake, D. A., Lacey, C. G., Benson,

A. J., Bower, R. G., \& Pimbblet, K. 2008, MNRAS, 386, 2145
Benoist, C., Maurogordato, S., da Costa, L. N., Cappi, A., \& Schaeffer, R. 1996, ApJ, 472, 452 
Benson, A. J., Cole, S., Frenk, C. S., Baugh, C. M., \& Lacey, C. G. 2000, MNRAS, 311, 793

Berlind, A. A. \& Weinberg, D. H. 2002, ApJ, 575, 587

Berlind, A. A., et al. 2003, ApJ, 593, 1

Bernardi, M., Nichol, R. C., Sheth, R. K., Miller, C. J., \& Brinkmann, J. 2006, AJ, 131, 1288

Blake, C., Collister, A., \& Lahav, O. 2008, MNRAS, 385, 1257

Blanton, M. R., et al. 2003, ApJ, 592, 819

Bower, R. G., Benson, A. J., Malbon, R., Helly, J. C., Frenk, C. S., Baugh, C. M., Cole, S., \& Lacey, C. G. 2006, MNRAS, 370,645

Brown, M. J. I., Webster, R. L., \& Boyle, B. J. 2000, MNRAS, 317,782

Brown, M. J. I., Zheng, Z., White, M., Dey, A., Jannuzi, B. T., et al. 2008, ApJ, 682, 937

Budavari, T., et al., 2003, ApJ, 595, 59

Bullock, J. S., Kolatt, T. S., Sigad, Y., Somerville, R. S., Klypin, A. A., Primack, J. R., Dekel, A. 2001, MNRAS, 321, 559

Bullock, J. S., Wechsler, R. H., \& Somerville, Rachel, S. 2002, MNRAS, 329, 246

Coil, A. L., Newman, J. A., Cooper, M. C., Davis, M., Faber, S. M., Koo, D. C., \& Willmer, C. N. A. 2006, ApJ, 644, 671

Coil, A. L., et al. 2008, ApJ, 672, 153

Colless, M., et al. 2001, MNRAS, 328, 1039

Conroy, C., Wechsler, R. H., \& Kravtsov, A. V. 2006, ApJ, 647, 201

Cooray, A. 2005a, MNRAS, 364, 303

Cooray, A. 2006, MNRAS, 365, 842

Davis, M., \& Geller, M. J. 1976, ApJ, 208, 13

Davis, M., Efstathiou, G., Frenk, C. S., \& White, S. D. M. 1985, ApJ, 292, 371

Davis, M., Meiksin, A., Strauss, M. A., da Costa, L. N., \& Yahil, A. 1988, ApJ, 333, L9

Dressler, A. 1980, ApJ, 236, 351

Eisenstein, D. J., et al. 2001, AJ, 122, 2267

Eisenstein, D. J., Blanton, M., Zehavi, I., Bahcall, N., Brinkmann, J., Loveday, J., Meiksin, A., \& Schneider, D. 2005, ApJ, 619, 178

Eisenstein, D. J., et al. 2005, ApJ, 633, 560

Gilks, W. R., Richardson, S., \& Spiegelhalter, D. J. 1996, Markov Chain Monte Carlo in Practice (London: Chapman and Hall)

Goto, T., Yamauchi, C., Fujita, Y., Okamura, S., Sekiguchi, M., Smail, I., Bernardi, M., \& Gomez, P. L. 2003, MNRAS346, 601

Guzzo, L., Strauss, M. A., Fisher, K. B., Giovanelli, R., \& Haynes, M. P. 1997, ApJ, 489, 37

Hamana, T., Yamada, T., Ouchi, M., Iwata, I., \& Kodama, T. 2006, MNRAS, 369, 1929

Hamilton, A. J. S. 1988, ApJ, 331, L59

Hartlap, J., Simon, P., \& Schneider, P. 2007, A\&A, 464, 399

Hawkins, E., et al. 2003, MNRAS, 346, 78

Ho, S., Lin, Y.-T., Spergel, D., \& Hirata, C. M. 2007, ArXiv Astrophysics e-prints, arXiv:0706.0727

Hubble, E.P. 1936, The Realm of the Nebulae (Oxford University Press: Oxford), 79

Jenkins, A., Frenk, C. S., White, S. D. M., Colberg, J. M., Cole, S., Evrard, A. E., Couchman, H. M. P., \& Yoshida, N. 2001, MNRAS, 321, 372

Jing, Y. P., Mo, H. J., \& Börner, G. 1998, ApJ, 494, 1

Jing, Y. P. \& Börner, G. 1998, ApJ, 503, 37

Jing, Y. P., Börner, G., \& Suto, Y. 2002, ApJ, 564, 15

Jing, Y. P., Suto, Y., \& Mo, H. J. 2007, ApJ, 657, 664

Koester, B. P., et al. 2007, ApJ, 660, 239

Kravtsov, A. V., Berlind, A. A., Wechsler, R. H., Klypin, A. A., Gottlöber, S., Allgood, B., \& Primack, J. R. 2004, ApJ, 609, 35

Kulkarni, G. V., Nichol, R. C., Sheth, R. K., Seo, H.-J., Eisenstein, D. J., \& Gray, A. 2007, MNRAS, 378, 1196

Lee, K., Giavalisco, M., Gnedin, O. Y., Somerville, R., Ferguson, H., Dickinson, M., \& Ouchi, M. 2006, ApJ, 642, 63

Loh, Y. S. 2003, Ph.D. thesis, Princeton Univ.

Loveday, J., Maddox, S. J., Efstathiou, G., \& Peterson, B. A. 1995, ApJ, 442, 457

Ma, C., \& Fry, J. N. 2000, ApJ, 543, 503

Madgwick, D. S. et al. 2003, MNRAS, 344, 847

Magliocchetti, M., \& Porciani, C. 2003, MNRAS, 346, 186

Mandelbaum, R., Seljak, U., Cool, R. J., Blanton, M., Hirata, C. M., \& Brinkmann, J. 2006, MNRAS, 372, 758

Masjedi, M., et al. 2006, ApJ, 644, 54
More, S., van den Bosch, F. C., Cacciato, M., Mo, H. J., Yang, X., \& Li, R. 2009, MNRAS, 392, 801

Moustakas, L. A., \& Somerville, R. S. 2002, ApJ, 577, 1

Navarro, J. F., Frenk, C. S., \& White, S. D. M. 1995, MNRAS, 275,56

Navarro, J. F., Frenk, C. S., \& White, S. D. M. 1996, ApJ, 462, 563

Navarro, J. F., Frenk, C. S., \& White, S. D. M. 1997, ApJ, 490, 493

Norberg, P., et al. 2001, MNRAS, 328, 64

Norberg, P., et al. 2002, MNRAS, 332, 827

Ouchi, M., et al. 2005, ApJ, 635, L117

Padmanabhan, N., White, M., Norberg, P., \& Porciani, C. 2009, MNRAS, 959

Park, C., Vogeley, M. S., Geller, M. J., \& Huchra, J. P. 1994, ApJ, 431, 569

Peacock, J. A., \& Smith, R. E. 2000, MNRAS, 318, 1144

Percival, W. J., et al. 2007, ApJ, 657, 645

Postman, M., \& Geller, M. J. 1984, ApJ, 281, 95

Press, W. H. \& Schechter, P. 1974, ApJ, 187, 425

Reid, B. A., \& Spergel, D. N. 2009, ApJ, 698, 143

Ross, N. P., Shanks, T., Cannon, R. D., Wake, D. A., Sharp, R. G., Croom, S. M., \& Peacock, J. A. 2008, MNRAS, 387, 1323

Schechter, P. 1976, ApJ, 203, 297

Scoccimarro, R., Sheth, R. K., Hui, L., \& Jain, B. 2001, ApJ, 546, 20

Scoccimarro, R., \& Sheth, R. K. 2002, MNRAS, 329, 629

Seljak, U. 2000, MNRAS, 318, 203

Seo, H.-J., Eisenstein, D. J., \& Zehavi, I. 2008, ApJ, 681, 998

Sheldon, E. S., et al. 2007, ArXiv e-prints, 709, arXiv:0709.1162

Sheth, R. K. \& Tormen, G. 1999, MNRAS, 308, 119

Simon, P., Hetterscheidt, M., Wolf, C., Meisenheimer, K., Hildebrandt, H., Schneider, P., Schirmer, M., \& Erben, T. 2008, arXiv:0805.3459

Springel, V., et al. 2005, Nature, 435, 629

Strauss, M. A., et al. 2002, AJ, 124, 1810

Tegmark, M., et al. 2006, Phys. Rev. D, 74, 123507

Tinker, J. L., Weinberg, D. H., Zheng, Z., \& Zehavi, I. 2005, ApJ, 631,41

Tinker, J. L., Norberg, P., Weinberg, D. H., \& Warren, M. S. 2007, ApJ, 659, 877

Tinker, J., Kravtsov, A. V., Klypin, A., Abazajian, K., Warren, M., Yepes, G., Gottlöber, S., \& Holz, D. E. 2008, ApJ, 688, 709

Vale, A., \& Ostriker, J. P. 2004, MNRAS, 353, 189

van den Bosch, Frank, C., Yang, X. H., \& Mo, H. J. 2003,

MNRAS, 340, 77

Wake, D. A., et al. 2008, MNRAS, 387, 1045

White, S. D. M., Tully, R. B., \& Davis, M. 1988, ApJ, 333, L45

White, M., Zheng, Z., Brown, M. J. I., Dey, A., \& Jannuzi, B. T. 2007, ApJ, 655, L69

Willmer, C. N. A., da Costa, L. N., \& Pellegrini, P. S. 1998, AJ, 115,869

Yan, R., Madgwick, D. S., \& White, M. 2003, ApJ, 598, 848

Yang, X. H., Mo, H. J., \& van den Bosch, F. C. 2003, MNRAS, 339,1057

Yang, X., Mo, H. J., Jing, Y. P., \& van den Bosch, F. C. 2005, MNRAS, 358, 217

Yoo, J., Tinker, J. L., Weinberg, D. H., Zheng, Z., Katz, N., \& Davé, R. 2006, ApJ, 652, 26

Yoo, J., Weinberg, D. H., Tinker, J. L., Zheng, Z., \& Warren, M. S. 2009, ApJ, 698, 967

York, D. G., et al. 2000, ApJ, 120, 1579

Zehavi, I., Blanton, M. R., Frieman, J. A., Weinberg, D. H., Mo, H. J., et al. 2002, ApJ, 571, 172

Zehavi, I., et al. 2004, ApJ, 608, 16

Zehavi, I., et al. 2005, ApJ, 621, 22

Zehavi, I., et al. 2005, ApJ, 630, 1

Zentner, A. R., Berlind, A. A., Bullock, J. S., Kravtsov, A. V., \& Wechsler, R. H. 2005, ApJ, 624, 505

Zheng, Z., Tinker, J. L., Weinberg, D. H., \& Berlind, A. A. 2002, ApJ, 575, 000

Zheng, Z. 2004, ApJ, 610, 61

Zheng, Z., et al. 2005, ApJ, 633, 791

Zheng, Z., \& Weinberg, D. H. 2007, ApJ, 659, 1

Zheng, Z., Coil, A. L., \& Zehavi, I. 2007, ApJ, 667, 760 
Zwicky, F., Herzog, E., Wild, P., Karpowicz, M., \& Kowal, C., 1961-1968, Catalog of Galaxies and of Clusters of Galaxies, Vols. 1-6, (Pasadena: California Institute of Technology) 